\title{
Forced Tunneling and Turning State Explosion in Pure Yang-Mills Theory
}

\author{
D. M. Ostrovsky ${ }^{1}$, G. W. Carter ${ }^{2}$, and E. V. Shuryak ${ }^{1}$ \\ ${ }^{1}$ Department of Physics and Astronomy, State University of New York, Stony Brook, NY 11794-3800 \\ ${ }^{2}$ Department of Physics, Box 351560, University of Washington, Seattle, WA 98195-1560
}

(18 April 2002)

\begin{abstract}
We consider forced tunneling in QCD, described semiclassically by instanton-antiinstanton field configurations. By separating topologically different minima we obtain details of the effective potential and study the turning states, which are similar to the sphaleron solution in electroweak theory. These states are alternatively derived as minima of the energy under the constraints of fixed size and Chern-Simons number. We study, both analytically and numerically, the subsequent evolution of such states by solving the classical Yang-Mills equations in real time, and find that the gauge field strength is quickly localized into an expanding shell of radiating gluons. The relevance to high-energy collisions of hadrons and nuclei is briefly discussed.
\end{abstract}

\section{INTRODUCTION}

\section{A. Instanton-Induced Scattering in QCD}

The existence of topologically distinct non-abelian gauge fields, with tunneling between corresponding classical vacua described semiclassically by instantons [i], is one of the most spectacular nonperturbative effects of field theory. Significant progress has been made in understanding instanton-induced effects in Quantum Chromodynamics (QCD), explaining both explicit $\mathrm{U}_{A}(1)$ chiral symmetry breaking at the single-instanton level [2] and spontaneous $\mathrm{SU}\left(N_{f}\right)$ chiral symmetry breaking by the instanton ensemble [3]. Euclidean correlation functions, studied phenomenologically and on the lattice, have been explained to a significant extent by instantons as well [ $⿴$.

With tunneling phenomena apparently so important in virtual quark and gluon propagation, it is reasonable to think them also relevant in real processes such as scattering or particle production in Minkowski space. We thus seek contributions to parton scattering amplitudes from the theory of instanton-related objects, and supporting experimental evidence.

With this as our motivation, we concentrate in this paper on the theoretical basis of such effects from pure Yang-Mills theory. Specific applications to high-energy processes with hadrons or nuclei are left for papers to follow, although we will discuss phenomenological generalities where relevant.

Progress in understanding of the role of tunneling in high energy processes has been tempered by technical problems for years. Significant insights were obtained in the 1980's [5] and further developed in the early 1990's [6,7] through work in electroweak theory. In this case, the instanton-induced cross section is readily identified by baryon number violation and many noteworthy features of these processes were found. However, quantitative estimates of the associated cross sections proved to be far below observable limits and interest quickly waned. Similar ideas have also been developed in QCD [8], notably the search for hard processes induced by small-sized instantons which continues at HERA [9].

Another role for instanton-induced processes has recently been proposed by Kharzeev, Kovchegov, and Levin [10] and Nowak, Shuryak, and Zahed [11]. These works focus on typical QCD instantons, of size $\rho \sim 1 / 3 \mathrm{fm}$ [3], which determine the semi-hard scale of $Q \sim 1-2$ $\mathrm{GeV}$. It was proposed that topological tunneling is behind the well-known features of high energy scattering described phenomenologically by the so-called "soft" pomeron. These ideas were further tested in Ref. [12], where they were demonstrated to be reasonably consistent with experimental data.

Since the 1960's attempts have been made to explain high-energy hadronic collisions with multi-peripheral models, with various ladder diagrams describing hadron production. It was realized that in order to get crosssections which are not falling at high energies, one needed vector field exchange in the $t$-channel. With the discovery of QCD, gluons naturally play this role. Generic pQCD-inspired models appeared with processes like that shown in Fig. 1(a). Eventually this development led to the BFKL gluon ladder [13], which produces an (approximately) supercritical pomeron, a "hard" pomeron with the intercept well above 1. Recent studies of high energy hard processes, especially at HERA, have indeed found strong growth of the cross section with energy for truly hard processes $\left(Q^{2} \gg 1 \mathrm{GeV}^{2}\right)$, consistent with the BFKL treatment.

But various data at the semi-hard scale of $Q^{2} \sim 1 \mathrm{GeV}^{2}$ demonstrate rather different growth with energy, consistent with a "soft" pomeron. Whatever it might be, the pomeron should be an object of a particular size deduced from the slope of its Regge trajectory, $\alpha^{\prime} \sim 1 /(2 \mathrm{GeV})^{2}$. This size of course cannot be explained by basically scaleinvariant pQCD, and thus calls for a nonperturbative derivation.

Existing models for the soft pomeron also include ladders made of $t$-channel gluons, and the differences between them lie mainly in the construction of their rungs. Each of the various models has a unique answer for what is actually produced in gluon-gluon partonic collisions. 
For example, in Ref. 115] a pair of pions in the scalar channel or a scalar glueball is produced. The introduction into this problem of instanton-induced vertices [16, 10, 11, , shown schematically in Fig. 1(b), led to a different idea: the object produced is neither a gluon (as in BFKL) nor any colorless hadronic state, but rather a colored cluster of the gluon field, which in turn decays into several gluons. It has been shown that the cross section peaks at an invariant cluster mass in the range $2.5-3 \mathrm{GeV}$ [10,11]. It is very important that the states which are produced are not a random group of gluons, but rather their coherent superposition. Understanding their composition is the main objective of this work.

A quantum-mechanical interpretation of the collision process is central to this question of prompt gluon production. An impressive body of work has addressed this problem with classical Weizsäcker-Williams fields of gluons, the Color Glass Condensate [14]. Here we consider a different classical process, one involving topological objects. In Fig. 11(c) we schematically show a barrier separating two topologically distinct classical vacua, with Chern-Simons numbers $N_{C S}=0$ and 1. Unlike a standard instanton transition, shown by the horizontal dashed line, in a high energy collision a finite amount of energy is absorbed. This can be viewed as a "forced tunneling" event (either of the other two dashed lines) which ends at a turning state, where the total energy is equal to the potential energy, so that the paths can exit the (Euclidean) domain below the barrier. These colored unstable objects are close relatives of electroweak sphalerons [17,18], which are defined at the barrier's peak. We will demonstrate how these objects then evolve with conserved energy, developing into an exploding shell of color field. This part of the process is diagrammed with the horizontal lines in Fig. 11(c).

Before we come to these explosions, we will discuss in detail the instanton-anti-instanton $(\bar{I} I)$ configurations which describe this forced tunneling. They provide one way toward the understanding of the effective potential separating topologically different gauge fields, as well as the turning states themselves. We then proceed to another derivation of the same results as static solutions in classical Yang-Mills theory constrained in size. The realtime decay of the static configuration is studied in detail, using both analytic and numerical methods, ultimately leading to a description of the expanding shells in terms of gluonic quanta.

\footnotetext{
${ }^{*}$ This will be introduced formally below. Here it is sufficient to note only that we consider a definite pair of gauge potentials, separated on one of the many coordinates of our quantum system.
}
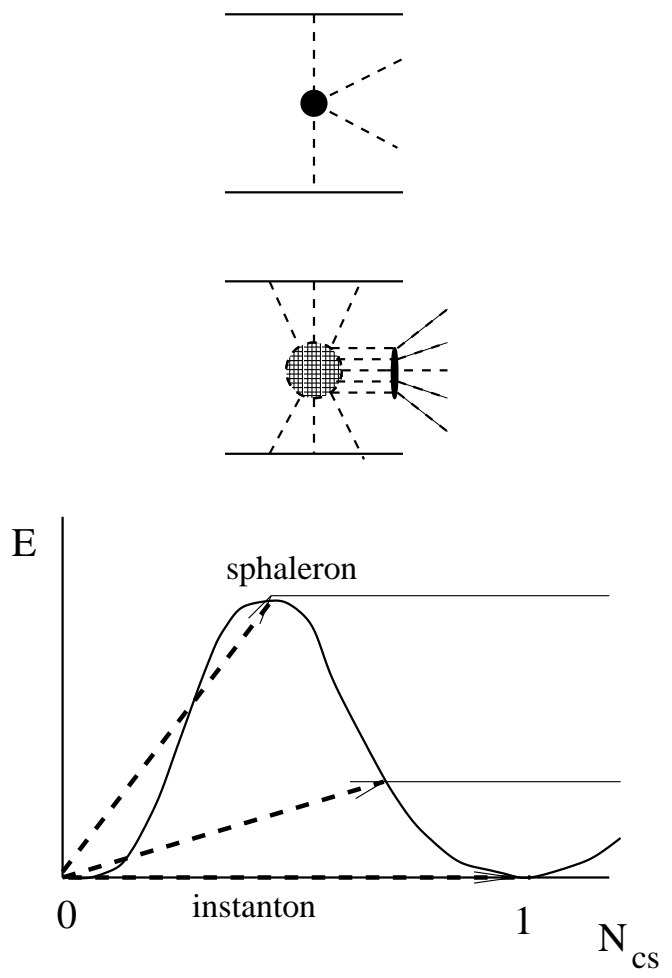

FIG. 1. The top of the figure compares (a) a typical inelastic perturbative process (two t-channel gluons collide, producing a pair of gluons) to (b) a nonperturbative inelastic process, incorporating collisions of a few $t$-channel gluons with the instanton (the shaded circle), resulting in multi-gluon production. The bottom figure (c) shows the same process, but in a quantum mechanical way. The energy of Yang-Mills field versus the Chern-Simons number, $N_{c s}$, is a periodic function, with zeros at integer points. The instanton (shown by the lowest dashed line) is a transition between such points. However if some nonzero energy is deposited into the process during transition, the virtual path (the dashed line) leads to a turning states, from which starts the real time motion outside the barrier (shown by horizontal solid lines). The maximal cross section corresponds to the transition to the top of the barrier, called a sphaleron.

\section{B. Spherically Symmetric Yang-Mills Fields}

For the $\mathrm{SU}(2)$ color subgroup in which we are interested, spherically symmetric field configurations of the gauge field $\mathcal{A}_{\mu}^{a}$ can be expressed through the following four space-time $(0, j=1 . .3)$ and color $(a=1 . .3)$ structures

$$
\begin{aligned}
\mathcal{A}_{j}^{a} & =A(r, t) \Theta_{j}^{a}+B(r, t) \Pi_{j}^{a}+C(r, t) \Sigma_{j}^{a} \\
\mathcal{A}_{0}^{a} & =D(r, t) \frac{x^{a}}{r}
\end{aligned}
$$

with

$$
\Theta_{j}^{a}=\frac{\epsilon_{j a m} x^{m}}{r}, \quad \Pi_{j}^{a}=\delta_{a j}-\frac{x_{a} x_{j}}{r^{2}}, \quad \Sigma_{j}^{a}=\frac{x_{a} x_{j}}{r^{2}} .
$$


It is convenient to express the scalar functions in Eq. (11) in terms of four $r$ and $t$ dependent functions, which are similar to the fields of the $1+1$ dimensional Abelian gauge-Higgs model $\left(A_{\mu=0,1}, \phi, \alpha\right)$ on a hyperboloid [19]:

$$
A=\frac{1+\phi \sin \alpha}{r}, \quad B=\frac{\phi \cos \alpha}{r}, \quad C=A_{1}, \quad D=A_{0} .
$$

One can express the field strengths in these terms as

$$
\begin{aligned}
\mathcal{E}_{j}^{a}=\mathcal{G}_{0 j}^{a}= & \frac{1}{r}\left[\partial_{0} \phi \sin \alpha+\phi \cos \alpha\left(\partial_{0} \alpha-A_{0}\right)\right] \Theta_{j}^{a} \\
& +\frac{1}{r}\left[\partial_{0} \phi \cos \alpha-\phi \sin \alpha\left(\partial_{0} \alpha-A_{0}\right)\right] \Pi_{j}^{a} \\
& +\left(\partial_{0} A_{1}-\partial_{1} A_{0}\right) \Sigma_{j}^{a}
\end{aligned}
$$

and

$$
\begin{aligned}
\mathcal{B}_{j}^{a}=\frac{1}{2} \epsilon_{j k l} \mathcal{G}_{k l}^{a}= & \frac{1}{r}\left[-\partial_{1} \phi \cos \alpha+\phi \sin \alpha\left(\partial_{1} \alpha-A_{1}\right)\right] \Theta_{j}^{a} \\
& +\frac{1}{r}\left[\partial_{1} \phi \sin \alpha+\phi \cos \alpha\left(\partial_{1} \alpha-A_{1}\right)\right] \Pi_{j}^{a} \\
& +\frac{1-\phi^{2}}{r^{2}} \Sigma_{j}^{a},
\end{aligned}
$$

where $\partial_{0} \equiv \partial_{t}$ and $\partial_{1} \equiv \partial_{r}$.

The action in $3+1$ dimensional Minkowski $(-,+,+,+)$ space reduces as

$$
\begin{aligned}
S= & \frac{1}{4 g^{2}} \int d^{3} x d t\left[\left(\mathcal{B}_{j}^{a}\right)^{2}-\left(\mathcal{E}_{j}^{a}\right)^{2}\right] \\
= & 4 \pi \int d r d t\left[\left(\partial_{\mu} \phi\right)^{2}+\phi^{2}\left(\partial_{\mu} \alpha-A_{\mu}\right)^{2}\right. \\
& \left.+\frac{\left(1-\phi^{2}\right)^{2}}{2 r^{2}}-\frac{r^{2}}{2}\left(\partial_{0} A_{1}-\partial_{1} A_{0}\right)^{2}\right],
\end{aligned}
$$

with the summation now over the $1+1$ dimensional $(-,+)$ metric.

The spherical ansatz is preserved by a set of gauge transformations generated by unitary matrices of the type

$$
U(r, t)=\exp \left(i \frac{\beta(r, t)}{2 r} \tau^{a} x^{a}\right)
$$

These transformations naturally coincide with the gauge symmetry of the corresponding abelian Higgs model:

$$
\phi^{\prime}=\phi, \quad \alpha^{\prime}=\alpha+\beta, \quad A_{\mu}^{\prime}=A_{\mu}+\partial_{\mu} \beta .
$$

This freedom can be used to gauge out, for example, the $\mathcal{A}_{0}$ component.

Topological properties of the gauge field are governed by the topological current

$$
K_{\mu}=-\frac{1}{32 \pi^{2}} \epsilon^{\mu \nu \rho \sigma}\left(\mathcal{G}_{\nu \rho}^{a} \mathcal{A}_{\sigma}^{a}-\frac{g}{3} \epsilon^{a b c} \mathcal{A}_{\nu}^{a} \mathcal{A}_{\rho}^{b} \mathcal{A}_{\sigma}^{c}\right) .
$$

Although this current is not gauge invariant, its change is related to the (gauge invariant) local topological charge

$$
\partial_{\mu} K^{\mu}=-\frac{1}{32 \pi^{2}} \mathcal{G}_{\mu \nu}^{a} \tilde{\mathcal{G}}_{\mu \nu}^{a} .
$$

Within the spherical ansatz and the $\mathcal{A}_{0}=0$ gauge the topological current takes a simpler form,

$$
\begin{aligned}
K^{0} & =\frac{1}{8 \pi^{2} r^{2}}\left[\left(1-\phi^{2}\right)\left(\partial_{1} \alpha-A_{1}\right)-\partial_{1}(\alpha-\phi \cos \alpha)\right] \\
K^{i} & =\frac{x^{i}}{8 \pi^{2} r^{3}}\left[\left(1-\phi^{2}\right) \partial_{0} \alpha-\partial_{0}(\alpha-\phi \cos \alpha)\right],
\end{aligned}
$$

while the topological charge becomes

$$
\begin{aligned}
\partial_{\mu} K^{\mu}= & \frac{1}{8 \pi^{2} r^{2}}\left\{-\partial_{0}\left[\left(1-\phi^{2}\right)\left(\partial_{1} \alpha-A_{1}\right)\right]\right. \\
& \left.+\partial_{1}\left[\left(1-\phi^{2}\right)\left(\partial_{0} \alpha-A_{0}\right)\right]\right\} .
\end{aligned}
$$

Note that only gauge-invariant combinations of field derivatives appear here.

As a "topological coordinate" marking the tunneling paths and the turning states one can use the ChernSimons number

$$
\begin{aligned}
N_{C S}=\int d^{3} x K_{0}= & -\frac{1}{2 \pi} \int d r\left(1-\phi^{2}\right)\left(\partial_{1} \alpha-A_{1}\right) \\
& +\left.\frac{1}{2 \pi}(\alpha-\cos \alpha)\right|_{r=0} ^{r=\infty}
\end{aligned}
$$

The first, gauge-invariant term is sometimes called the corrected or true Chern-Simons number [20,21], $\tilde{N}_{C S}$, while the second (gauge-dependent) term is referred to as the winding number. It is the change in $\tilde{N}_{C S}$ which is equivalent to the integral over the local topological charge.

\section{INSTANTON-ANTIINSTANTON CONFIGURATIONS}

\section{A. Forced Tunneling}

A brief introduction to the quantum mechanics of gluons in high energy collisions has been given in the introduction. The effect of colliding partons can be included in various forms. For example, these fields can be represented as non-zero external currents which affect the tunneling paths of Yang-Mills field. In the zero-current, vacuum case, the usual instanton solutions are spherically symmetric in four Euclidean dimensions. The collision problem of two (or more) partons, on the contrary, at non-zero impact parameters does not have even an axial symmetry. The reader therefore may wonder why this (and all previous works) on the subject consider $3+1$ dimensional spherically symmetric fields.

The justification for this ansatz is that the absolute magnitude of the tunneling field is large compared to external forces. Also, as will be shown below, spherically 
symmetric clusters are an energy minimum for fixed size and topological coordinate. Should the resulting cluster not have exact spherical symmetry one can always approach the problem perturbatively, considering first the external forces projected onto the direction of tunneling, and then other components as small corrections. The resulting $1+1$ dimensional problem is readily solved numerically and, to a great extent, analytically.

Unlike separated instantons $(I)$ and antiinstantons $(\bar{I})$, combined $\bar{I} I$ configurations are neither selfdual nor antiselfdual and do not satisfy classical equations of motion. They are not extrema of the action, since they describe the valley stretching between true extrema - the zero field (equivalent to an $\bar{I} I$ at zero separation) and wellseparated $\bar{I} I$ pair. Substituting any $\bar{I} I$ trial function into the of Yang-Mills equation of motion, we find a finite

$$
D_{\mu} \mathcal{G}_{\mu \nu}=J_{\nu}
$$

This means some external current must be applied to the gauge fields if we want to use semiclassical analysis. The process can only then be interpreted as a classical $\bar{I} I$, or a forced path. There are two interpretations of $\bar{I} I$ configurations with different consequences.

The historical view is that such fields describe quantum fluctuations in the Yang-Mills vacuum, the process in which a virtual path goes under the barrier, then reverses course and ends up in the same minimum from which it started. This process has zero net topological charge. Naturally, the early studies concentrated on the action corresponding to these configurations, the quantity which controls its weight in the path integral. The first such work was done long ago by Callan, Dashen, and Gross [22], resulting in a dipole force and the action $\delta S \sim 1 / T^{4}$ at large distance $T$ between the centers. Higher terms in the multipole expansion have been discussed in literature after that, e.g. 23]. When it was eventually realized that quark-induced $I I$ pairings are more important for the instanton ensemble in QCD [24], interest in the pure Yang-Mills theory waned.

In this paper we will however take a different view of $\bar{I} I$ configurations. Since the external forces from the partonic current do work on the $\bar{I} I$ pair, the energy at intermediate times is non-zero. We will consider only cases in which the fields at positive and negative times are essentially the same (modulo a sign and, sometimes, a gauge transformation). Thus this energy will be even under $t \rightarrow-t$, with a natural maximum at $t=0$. As a result, all quantities which are odd under this transformation (like the electric field) naturally vanish at this instant. The remaining, purely magnetic configuration is what we define as the turning state of this path.

The resulting action corresponds to an excitation probability of this turning state created by the external current $J$,

$$
P \sim \mid\left.\langle 0|J| \text { turning state }\rangle\right|^{2}
$$

Through this mechanism the excitation of $\bar{I} I$ pairs leads to the production of real particles, as advertized in the Introduction and to be analyzed in the next sections.

\section{B. Simple $\bar{I} I$ Trial Functions}

We now consider the simplest example of a possible turning state, a straightforward $\bar{I} I$ sum ansatz. With it, we can demonstrate some basic features, although we will find them insufficient for our purposes and move to a more complicated ansatz in the next subsection.

Written the singular gauge, the sum ansatz is:

$$
\frac{g}{2} \mathcal{A}_{a \mu}^{\text {sum }}(x)=\frac{\bar{\eta}_{a \mu \nu} y_{1}^{\nu} \rho^{2}}{y_{1}^{2}\left(y_{1}^{2}+\rho^{2}\right)}+\frac{\eta_{a \mu \nu} y_{2}^{\nu} \rho^{2}}{y_{2}^{2}\left(y_{2}^{2}+\rho^{2}\right)},
$$

where we assume that both the instanton and the antiinstanton (the first and second terms, respectively) have the same color orientation and size $\rho$. The vectors $y_{1}=x-z_{I}$ and $y_{2}=x-z_{\bar{I}}$ are the distances from the observation point $x$ to the instanton and antiinstanton centers. In what follows we assume $z_{I}=(T / 2,0,0,0)$ and $z_{\bar{I}}=(-T / 2,0,0,0)$, where the imaginary time between centers is $T$.

Note that although a single instanton's profile behaves as $1 / x$ near the origin, the physical quantity $\left(\mathcal{G}_{\mu \nu}^{a}\right)^{2}$ is finite. However, for the sum ansatz this feature is lost and the same quantity goes as $1 / x^{2}$ near the origin.

This unphysical feature can be quickly remedied by the ratio ansatz 25], which for identical sizes and orientations is

$$
\frac{g}{2} \mathcal{A}_{a \mu}^{\text {ratio }}(x)=\frac{\eta_{a, \mu \nu} y_{1}^{\nu} \frac{\rho^{2}}{y_{1}^{2}}+\bar{\eta}_{a, \mu \nu} y_{2}^{\nu} \frac{\rho^{2}}{y_{2}^{2}}}{1+\frac{\rho^{2}}{y_{1}^{2}}+\frac{\rho^{2}}{y_{2}^{2}}}
$$

These trial functions are simple enough to have analytic expressions for the field strength, the energy of static turning states, and the Chern-Simons number. For reference, one has the following expressions for the magnetic and electric fields squared:

$$
\begin{aligned}
\overrightarrow{\mathcal{B}}^{2}= & 16384\left(768 t^{8}+1024 r^{2} t^{6}+3072 t^{6}+2304 t^{6} R^{2}\right. \\
& +6400 r^{2} t^{4} R^{2}+2048 r^{2} t^{4}+512 r^{4} t^{4}+1824 t^{4} R^{4} \\
& +3072 t^{4}+4608 t^{4} R^{2}+1024 r^{6} t^{2}+192 t^{2} R^{4} \\
& +512 r^{2} t^{2} R^{2}-1024 r^{4} t^{2}+144 t^{2} R^{6}+1216 r^{2} t^{2} R^{4} \\
& +2816 r^{4} t^{2} R^{2}+288 r^{4} R^{4}+768 r^{8}+768 r^{6} R^{2} \\
& \left.+48 r^{2} R^{6}+3 R^{8}\right) /\left(16 r^{4}+32 r^{2} t^{2}+8 r^{2} R^{2}+16 t^{4}\right. \\
& \left.-8 t^{2} R^{2}+R^{4}+32 r^{2}+32 t^{2}+8 R^{2}\right)^{4}, \\
& \overrightarrow{\mathcal{E}}^{2}=1048576 t^{2}\left(32 r^{2} t^{4}+48 t^{4} R^{2}+64 r^{4} t^{2}+64 r^{2} t^{2}\right. \\
& +80 r^{2} t^{2} R^{2}+48 t^{2} R^{2}+24 t^{2} R^{4}+12 R^{4}+32 r^{2} \\
& +32 r^{6}+64 r^{2} R^{2}+26 r^{2} R^{4}+64 r^{4} R^{2} \\
& \left.+64 r^{4}+12 R^{2}+3 R^{6}\right) /\left(16 r^{4}+32 r^{2} t^{2}+8 r^{2} R^{2}\right. \\
& \left.+16 t^{4}-8 t^{2} R^{2}+R^{4}+32 r^{2}+32 t^{2}+8 R^{2}\right)^{4} .
\end{aligned}
$$


Their scalar product is

$$
\begin{aligned}
\overrightarrow{\mathcal{B}} \cdot \overrightarrow{\mathcal{E}}= & -393216 t R\left(R^{2}+2+4 r^{2}+4 t^{2}\right)\left(16 t^{4}\right. \\
& +24 t^{2} R^{2}+32 r^{2} t^{2}+32 t^{2}+R^{4}+16 r^{4} \\
& \left.+8 r^{2} R^{2}\right) /\left(16 r^{4}+32 r^{2} t^{2}+8 r^{2} R^{2}+16 t^{4}\right. \\
& \left.-8 t^{2} R^{2}+R^{4}+32 r^{2}+32 t^{2}+8 R^{2}\right)^{4},
\end{aligned}
$$

where we have set $\rho=1$ and $R=T$ is the intercenter distance.

One can see that, in the simplest case of identical sizes and orientations for the $I$ and $\bar{I}$, time reflection symmetry $t \rightarrow-t$ of the problem is indeed manifest, so that

$$
\mathcal{A}_{0}^{a}(\vec{r}, t=0)=0, \quad \mathcal{E}_{m}^{a}(\vec{x}, t=0)=0 .
$$

This is illustrated in Fig. 2(b). Since configurations of this type interpolate between a mostly dual region, with $\mathcal{E}_{m}^{a}\left(z_{I}\right)=\mathcal{B}_{m}^{a}\left(z_{I}\right)$, to an anti-dual region, where $\mathcal{E}_{m}^{a}\left(z_{\bar{I}}\right)=$ $-\mathcal{B}_{m}^{a}\left(z_{\bar{I}}\right)$, it is intuitive that the electric field vanishes in the center.

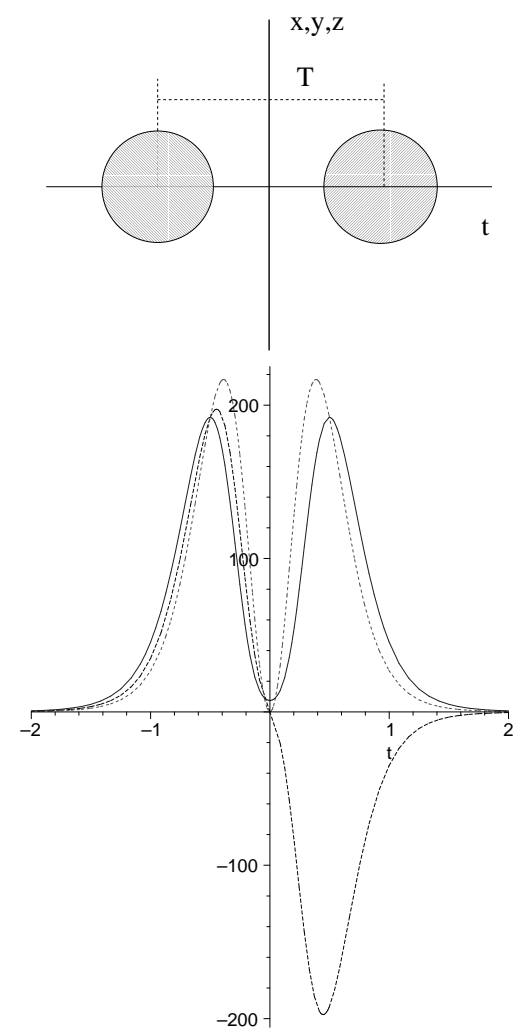

FIG. 2. Instanton-antiinstanton configurations. (a) A schematic picture in Euclidean space-time. The thick vertical line, $t=0$, corresponds to the location of the turning state. The definition of the inter-center distance $T$ is also shown. (b) Distribution along the time axis of $2 \overrightarrow{\mathcal{B}}^{2}, 2 \overrightarrow{\mathcal{E}}^{2}$, and $2 \overrightarrow{\mathcal{B}} \cdot \overrightarrow{\mathcal{E}}$ for the ratio ansatz with $T=\rho$, shown by the solid, dashed, and short-dashed lines respectively. The curve for $\overrightarrow{\mathcal{B}} \cdot \overrightarrow{\mathcal{E}}$ is the only one which is $t$-odd.
This situation can be readily interpreted in the $\mathcal{A}_{0}=0$ gauge, in which the electric field is simply the time derivative of the gauge field - the canonical momentum in Yang-Mills field quantization. Thus the $t=0$ magnetic state is indeed identified as a turning state, in which motion is momentarily stopped. For separation $T$ comparable to the size $\rho$ the energy is finite, with a maximum $E \sim 1 /(g \rho)$.

The energy $E$ and Chern-Simons number $N_{C S}$ for either the sum or ratio ansatz can be calculated as a function of separation $T$ directly, with the hope that a parametric plot of $E\left(N_{C S}\right)$ will reveal a useful profile of the barrier as a function of this topological coordinate.

Alas, for the sum ansatz this idea produces reasonable results only for very large separation, $T \geq 2 \rho$. When $T$ is of the order $\rho$, the energy $E(T)$ of the turning state (as well as the action for the entire configuration) becomes very large, while the topological coordinate $N_{C S}(T)$ remains fixed. It is therefore obvious that this set of paths does not describe the travel across the ridge separating classical vacua which we want to study. Instead, this path rises with the barrier but continues to increase as the origin is approached, following a direction apparently orthogonal to the topological coordinate we want to study.

The ratio ansatz yields somewhat better results, with finite (and even simple) field structure at all $T$, including the point $T=0$. However the results, shown in Fig. 3, indicate that this set of trial functions can only accomplish about one third of the journey we would like to make, in terms of the topological quantity $N_{C S}$. This inadequacy will become apparent after comparison with the results to follow.

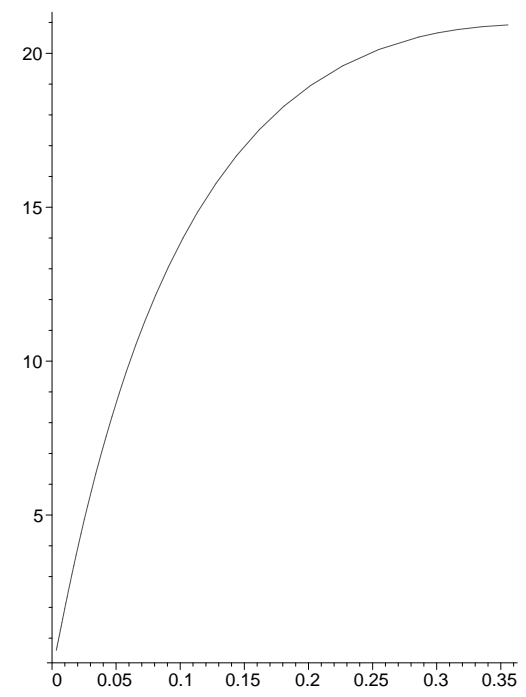

FIG. 3. The normalized energy, ER, versus the Chern-Simons number for the ratio ansatz. 


\section{The Yung Ansatz, or Going Uphill}

As a natural set of $\bar{I} I$ configurations, one of us 26] suggested starting from a well-separated pair and going downhill, along the gradient of the actioni. Naturally, minimization of the action leads to complete $\bar{I} I$ annihilation and zero action.

It was shown by Yung that these configuration can generally be obtained from a solution of the streamline equation [23]. He found solutions for large separation $T \gg \rho$ and used them to derive the next order terms in the $\bar{I} I$ interaction, to $O\left(1 / T^{6}\right)$. A clever conformal symmetry was used to reduce the Yang-Mills problem to that of a double well potential. The same trick was then used in the numerical solution of the streamline equation [7.27], in which it was observed that the approximate ansatz suggested by Yung also happens to be a very accurate approximation to true solution, not only at large $T$ (as Yung intended) but in fact for all finite $\bar{I} I$ separations $T$. As expected, at $T=0 \bar{I} I$ annihilation occurs and the field strength vanishesf.

Since we take a different view of $\bar{I} I$ configurations in this work, we interpret a solution of the streamline equation (or Yung ansatz) as a set of forced paths going uphill against the gradient of the force. This process reaches its turning point (or state), with some maximal energy and Chern-Simons number, and then turns back. Because the process proceeds uphill, unlike with other trial functions with some arbitrary driving force, we expect that all trajectories rise along the same path, although those with larger $T$ go further up.

The Yung ansatz for the field configuration is rather complicated, and is best written in matrix form:

$$
\begin{aligned}
i g \mathcal{A}_{\mu}^{Y \text { ung }}(x)= & i g \mathcal{A}_{a \mu}^{Y \text { ung }}(x) \frac{\tau^{a}}{2} \\
= & \frac{\bar{y}_{2}}{\sqrt{\tilde{y}_{2}}} \frac{R}{\sqrt{R^{2}}} \frac{\left(\bar{\sigma}_{\mu} y_{1}-y_{1}^{\mu}\right) \rho_{1}^{2}}{y_{1}^{2}\left(y_{1}^{2}+\rho_{1}^{2}\right)} \frac{\bar{R}}{\sqrt{R^{2}}} \frac{\bar{y}_{2}}{\sqrt{\tilde{y}_{2}}} \\
& +\frac{\left(\bar{\sigma}_{\mu} y_{2}-y_{2}^{\mu}\right) \rho_{2}^{2}}{y_{2}^{2}+\rho_{2}^{2}}+\frac{\rho_{1} \rho_{2}}{z y_{1}^{2}\left(y_{2}^{2}+\rho_{2}^{2}\right)} \\
& {\left[\left(\bar{\sigma}_{\mu} y_{1}-y_{1}^{\mu}\right)-\frac{\bar{y}_{2}}{\sqrt{\tilde{y}_{2}}}\right.} \\
& \left.\frac{R}{\sqrt{R^{2}}}\left(\bar{\sigma}_{\mu} y_{1}-y_{1}^{\mu}\right) \frac{\bar{R}}{\sqrt{R^{2}}} \frac{\overline{\tilde{y}}_{2}}{\sqrt{\tilde{y}_{2}}}\right],
\end{aligned}
$$

\footnotetext{
$\dagger$ This can easily be done numerically, and a set of such curves for the quantum-mechanical double well potential and the corresponding set of $\bar{I} I$ configurations was found in that work.

$¥$ This is not obvious from the Yung expression; it was first found numerically. The Yung formula's complicated result at $T=0$ is nothing but a pure gauge.
}

where $z$ is related to the conformal-invariant distance, $\left(R^{2}+\rho_{1}^{2}+\rho_{2}^{2}\right) /\left(\rho_{1} \rho_{2}\right)$. In the case $\rho_{1}=\rho_{2}=\rho$, which is the only one we need, this relation reads

$$
z^{2}=\frac{R^{2}+2 \rho^{2}+\sqrt{\left(R^{2}+2 \rho^{2}\right)^{2}-4 \rho^{2}}}{2 \rho^{2}} .
$$

All vectors without an indicative index are $\mathrm{SU}(2)$ matrices obtained by their contraction with the vector $\sigma_{\mu}=$ $(1,-i \vec{\tau})$, for example $R=x_{1}-x_{2}=R_{\mu} \sigma_{\mu}$. An overbar similarly denotes contraction with $\bar{\sigma}=(1, i \vec{\tau})$. Note that barred and unbarred matrices always alternate, in all terms; this is because one index of each matrix is dotted and the other not, in spinor notation. Finally, the additional coordinate is

$$
\tilde{y}_{2}=x_{2}-\frac{R \rho_{2}}{z \rho_{1}-\rho_{2}} .
$$

Note that the first term is the instanton in the singular gauge, the second is the anti-instanton in the regular gauge, and the third is a "correction" term. The benefit of this representation is that the same 't Hooft symbol appears in all three terms, and the entire construction originates from conformal transformation of a spherically symmetric configuration in which $\bar{I}, I$ share the same center. An unfortunate feature of this expression is that time-reversal symmetry is far from obvious, and it is not clear that the electric field at the mid-plane vanishes. However, this is in fact the case and the field at $t=0$ can be interpreted as a turning state.

Although all three trial functions are similar at large $\bar{I} I$ separation $T$, they are drastically different at $T \sim \rho$. The Yung ansatz is the only one which allows us to reasonably study the effects of a large change in topological number. The variation of the Chern-Simons number of the turning state $(t=0)$ as a function of the $\bar{I} I$ separation $T$ can be seen in Fig. 1 . In this case we scan the entire range $[0,1]$.

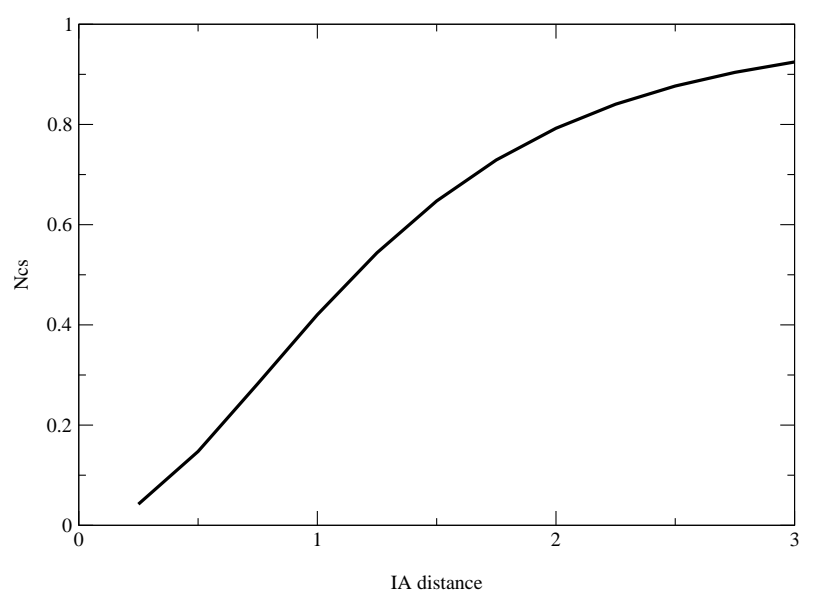

FIG. 4. $\quad N_{C S}$ versus the distance between $\bar{I} I$ centers $T$ in the Yung ansatz. 
We now proceed with a more detailed study of the static turning states, residing on the $t=03$-plane. The simplest observable is the shape of the corresponding magnetic field squared, or the energy density distribution, shown in Fig. 5 for few selected values of $\bar{I} I$ distance $T$. Note that the curve for $T=2$ (the most like the sphaleron) show indeed the largest magnitude of the magnetic field. The shape is however rather uniform. Note also that, unlike the case of the faulty sum and ratio trial functions, for smaller $T$ the field strength decreases, ultimately disappearing at $T=0$.

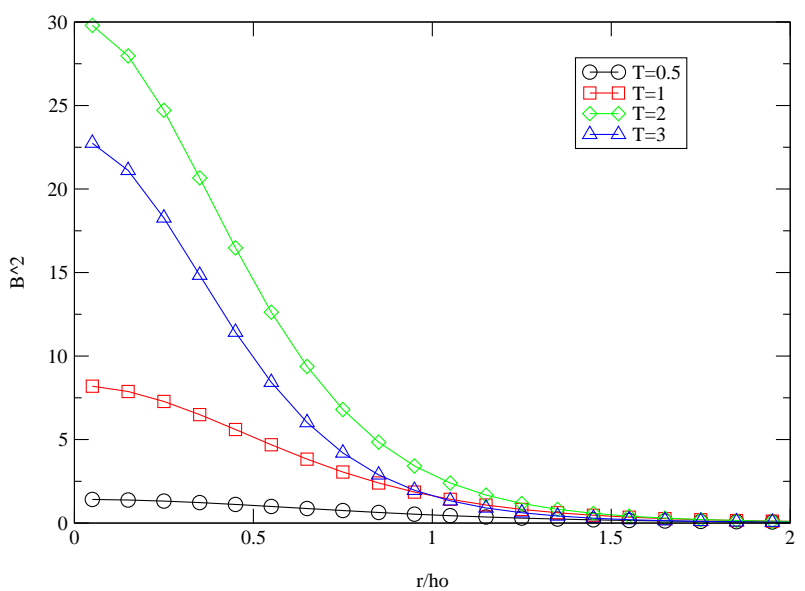

FIG. 5. The $\mathcal{B}(r)^{2}$ profile, not normalized, for the four values of the $\bar{I} I$ distance $T$ (in units of $\rho$ ) indicated in the legend.

The energy and energy density of the turning state configurations is therefore rather different for different $T$.

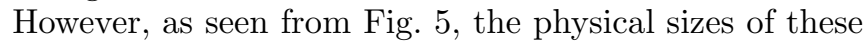
objects are different as well. As classic Yang-Mills theory has scale invariance, one may wish to make the more natural comparison of a scale-invariant combination, the energy times the r.m.s. radius, $R$, defined as

$$
R^{2}=\frac{\int d^{3} r r^{2} \mathcal{B}^{2}}{\int d^{3} r \mathcal{B}^{2}}
$$

In these terms, the normalized energy is

$$
E R=\frac{1}{2}\left[\int d^{3} r r^{2} \mathcal{B}^{2} \times \int d^{3} r \mathcal{B}^{2}\right]^{1 / 2} .
$$

This quantity is plotted versus the topological charge difference in Fig. 6, and indeed displays a parabolic-looking maximum near $N_{C S}=1 / 2$.
Energy*Size versus Ncs

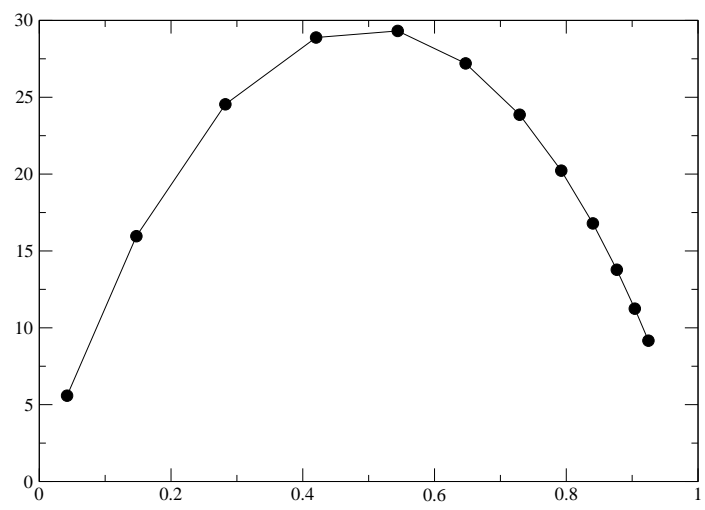

Time dependent paths: $\mathrm{E}^{*} \mathrm{R}$ vs Ncs

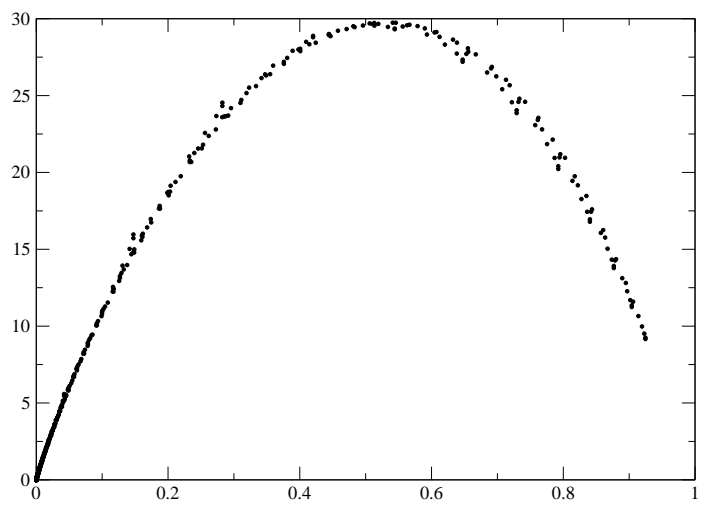

FIG. 6. The normalized energy, ER, versus the Chern-Simons number for the Yung ansatz. Plot (a) shows the positions of the turning states for various $T$, while (b) combines many points along the path $(t \neq 0)$; their small spread means that Yung ansatz is nearly going directly uphill, thus passing via the same points for different $T$.

Instead of only looking at the static $t=0$ (and zero electric field) turning states, one can instead follow the (scale invariant) energy $E R$ and the Chern-Simons number as a function of time $t$ along each each path. As expected, all the paths in Fig. 6(b), for any $T$, actually climb nearly exactly the same cliff, as they propagate into larger values of our topological coordinate.

\section{TURNING STATES FROM CONSTRAINED MINIMIZATION}

We will now define turning states in terms of the gauge field, which connect the Euclidean and Minkowski domains of the field's path. The turning state is characterized by the condition that the generalized momentum, which in the $\mathcal{A}_{0}=0$ gauge coincides with the chromoelectric field, vanishes or, equivalently, that all first time derivatives of the spatial field components are zero. Using the notation introduced in Section IB, this in turn 
means that $\partial_{0} \phi=\partial_{0} \alpha=\partial_{0} A_{1}=0$ at the time when the transition occurs. From now on we assume that moment to be $t=0$.

At any given time it is possible to use the special gauge transformation, Eq. (7), with a time-independent angle $\beta$ to gauge out $A_{1}(r)$, still within the $A_{0}=0$ gauge. At $t=0$ the energy of the field can thus be written

$$
E=\frac{4 \pi}{g^{2}} \int d r\left[\left(\partial_{r} \phi\right)^{2}+\phi^{2}\left(\partial_{r} \alpha\right)^{2}+\frac{\left(1-\phi^{2}\right)^{2}}{2 r^{2}}\right]
$$

We now address the question of the minimal potential energy of static Yang-Mills field, consistent with the appropriate constraints: (i) a fixed value of the (corrected) Chern-Simons number, and (ii) a given value of the r.m.s. size. The former parametrizes the position of configuration on the topological scale, and the latter is needed to break dilatation symmetry of the problem, which otherwise prevents any configuration of finite size from being the minimum of the energy.

We will break the scale invariance of the theory by setting a requirement that the ratio

$$
\left\langle r^{2}\right\rangle=\frac{\int d^{3} x r^{2} B^{2}}{\int d^{3} x B^{2}}
$$

has a particular value, $\rho^{2}$, for the static solution we seek. To keep both the Chern-Simons number and mean radius constant we introduce Lagrange multipliers and search for their minimal combination of

$$
\begin{aligned}
\tilde{E}= & \frac{4 \pi}{g^{2}} \int d r\left(1+\frac{r^{2}}{\rho^{2}}\right)\left[\left(\partial_{r} \phi\right)^{2}+\phi^{2}\left(\partial_{r} \alpha\right)^{2}+\frac{\left(1-\phi^{2}\right)^{2}}{2 r^{2}}\right] \\
& +\frac{\eta}{2 \pi} \int d r\left(1-\phi^{2}\right) \partial_{r} \alpha,
\end{aligned}
$$

where the tilde denotes the constrained energy. It is convenient to introduce a dimensionless variable,

$$
\xi=2 \arctan \left(\frac{r}{\rho}\right)-\frac{\pi}{2}
$$

so that

$$
\begin{gathered}
\tilde{E}=\frac{8 \pi}{g^{2}} \int_{-\pi / 2}^{\pi / 2} d \xi\left[\left(\partial_{\xi} \phi\right)^{2}+\phi^{2}\left(\partial_{\xi} \alpha\right)^{2}+\frac{\left(1-\phi^{2}\right)^{2}}{2 \cos ^{2} \xi}\right. \\
\left.+\kappa\left(1-\phi^{2}\right) \partial_{\xi} \alpha\right]
\end{gathered}
$$

where $\kappa=\eta \rho g^{2} /\left(32 \pi^{2}\right)$.

\footnotetext{
$\S$ Without the term introduced to fix the Chern-Simons number or, equivalently, for zero corresponding Lagrange multiplier, this problem would be equivalent to the $\mathrm{SU}(2)$ sphaleron on a 3 -d sphere that was solved by Smilga 28.
}

The Euler-Lagrange equations for the remaining fields are

$$
\partial_{\xi}^{2} \phi-\phi\left(\partial_{\xi} \alpha\right)^{2}+\frac{(1-\phi)^{2} \phi}{\cos ^{2} \xi}+2 \kappa \phi \partial_{\xi} \alpha=0
$$

and

$$
\partial_{\xi}\left(\phi^{2} \partial_{\xi} \alpha\right)+\kappa \partial_{\xi}\left(1-\phi^{2}\right)=0
$$

Finiteness of the energy requires the boundary conditions $\phi^{2}(-\pi / 2)=\phi^{2}(\pi / 2)=1$. Eq. (32) integrates to

$$
\partial_{\xi} \alpha=-\kappa \frac{1-\phi^{2}}{\phi^{2}}
$$

with a vanishing integration constant as follows from the form of the energy. After the substitution of $\partial_{\xi} \alpha$ into Eq. (31) one has

$$
\partial_{\xi}^{2} \phi+\frac{\left(1-\phi^{2}\right) \phi}{\cos ^{2} \xi}=\kappa^{2} \frac{1-\phi^{4}}{\phi^{3}}
$$

A solution to this equation exists for $-1<\kappa<1$,

$$
\phi^{2}=1-\left(1-\kappa^{2}\right) \cos ^{2} \xi
$$

Hereafter we assume that $\phi$ is positive.

In term of the usual $r$ coordinate, we have instead

$$
\begin{aligned}
\phi(r) & =\left(1-\left(1-\kappa^{2}\right) \frac{4 \rho^{2} r^{2}}{\left(r^{2}+\rho^{2}\right)^{2}}\right)^{1 / 2} \\
\partial_{r} \alpha(r) & =-2 \kappa \frac{1-\phi^{2}}{\phi^{2}} \frac{\rho}{r^{2}+\rho^{2}} .
\end{aligned}
$$

The sphaleron solution corresponds to $\kappa=0$ and

$$
\phi(r)=\frac{\left|r^{2}-\rho^{2}\right|}{r^{2}+\rho^{2}}, \quad \alpha(r)=\pi \theta(r-\rho) .
$$

For any $\kappa$ and mean squared radius $\left\langle r^{2}\right\rangle=\rho^{2}$, the potential energy density is

$$
\frac{1}{2} B^{2}=24 \rho^{4} \frac{\left(1-\kappa^{2}\right)^{2}}{\left(r^{2}+\rho^{2}\right)^{4}}
$$

the integral of which is the potential (magnetic) energy of the static configuration,

$$
E_{B}=3 \pi^{2} \frac{\left(1-\kappa^{2}\right)^{2}}{g^{2} \rho} .
$$

The corrected Chern-Simons number, computed from the first term of Eq. (13), is

$$
\tilde{N}_{C S}=\frac{1}{4} \operatorname{sign}(\kappa)(2+|\kappa|)(1-|\kappa|)^{2} .
$$

Figure 7 shows the profile of the potential energy $E_{B}$ versus $N_{C S}$. It is very similar, although not identical, to the findings of the preceding section (see Fig. 6) where Yung's ansatz was used for forced paths. 


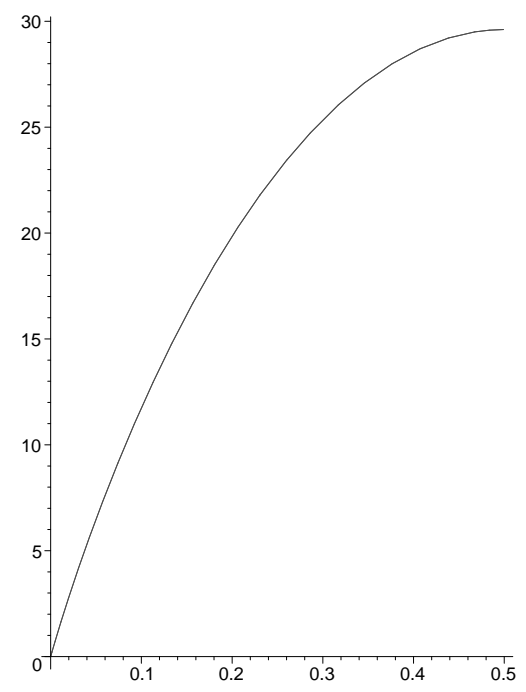

FIG. 7. The potential energy $E_{B}$ versus $\tilde{N}_{C S}$, for the analytic turning state solution of Eq. (36).

\section{EXPLOSIONS OF THE TURNING STATES: ANALYTIC TREATMENT}

We are now going to use the static field configuration, found in previous section, as an initial condition for realtime, Minkowski evolution of the gauge field. Let us first consider the equations of motion in the $1+1$ dimensional dynamical system. Variation of the action, Eq. (6), gives

$$
\begin{aligned}
\partial_{\mu} \partial^{\mu} \phi+\phi\left(\partial_{\mu} \alpha-A_{\mu}\right)^{2}+\frac{\left(1-\phi^{2}\right) \phi}{r^{2}} & =0 \\
\partial^{\mu}\left[\phi^{2}\left(\partial_{\mu} \alpha-A_{\mu}\right)\right] & =0 \\
\phi^{2}\left(\partial_{1} \alpha-A_{1}\right)-\partial_{0}\left[\frac{r^{2}}{2}\left(\partial_{0} A_{1}-\partial_{1} A_{0}\right)\right] & =0 \\
\phi^{2}\left(\partial_{0} \alpha-A_{0}\right)-\partial_{1}\left[\frac{r^{2}}{2}\left(\partial_{0} A_{1}-\partial_{1} A_{0}\right)\right] & =0 .
\end{aligned}
$$

The solution of Eq. (42) has the form

$$
\begin{aligned}
& \phi^{2}\left(\partial_{0} \alpha-A_{0}\right)=-\partial_{1} \psi \\
& \phi^{2}\left(\partial_{1} \alpha-A_{1}\right)=-\partial_{0} \psi,
\end{aligned}
$$

where $\psi(r, t)$ is an arbitrary smooth function. Eqs. (43) are consistent with this solution if

$$
\partial_{0} A_{1}-\partial_{1} A_{0}=-\frac{2 \psi}{r^{2}}
$$

Now, combining Eq. (42) and Eqs. (43) one has

$$
\partial^{\mu}\left(\frac{\partial_{\mu} \psi}{\phi^{2}}\right)=\partial_{0} A_{1}-\partial_{1} A_{0}=\frac{2 \psi}{r^{2}}
$$

which can be viewed as a necessary and sufficient condition for $\psi$ to be a solution for Eq. (42) and Eqs. (43) simultaneously. Eq. (41) is now

$$
\partial_{\mu} \partial^{\mu} \phi-\frac{\left(\partial_{\mu} \psi\right)^{2}}{\phi^{3}}+\frac{\left(1-\phi^{2}\right) \phi}{r^{2}}=0 .
$$

The initial conditions for Eqs. (46) and (47) are

$$
\begin{aligned}
\phi(r, 0) & =\phi(r), \\
\left.\partial_{0} \phi(r, t)\right|_{t=0} & =0, \\
\partial_{1} \psi(r, 0) & =-\phi(r)^{2} \partial_{0} \alpha(r)=0 \Rightarrow \psi(r, 0)=0, \\
\left.\partial_{0} \psi(r, t)\right|_{t=0} & =-\phi(r)^{2} \partial_{1} \alpha(r),
\end{aligned}
$$

where the $t$-independent fields on the right sides of the equations are the static solutions of $\phi$ and $\alpha$ from the previous section.

As with static solutions, it is more convenient to discuss the time-evolution equations in hyperbolic coordinates. Let us choose $\omega$ and $\tau$ such that

$$
r=\frac{\rho \cos \omega}{\cos \tau-\sin \omega}, \quad t=\frac{\rho \sin \tau}{\cos \tau-\sin \omega} .
$$

The physical domain of $0<r<\infty$ and $-\infty<t<\infty$ is covered by $-\pi / 2<\omega<\pi / 2$ and $-\pi / 2+\omega<\tau<\pi / 2-\omega$. For $t>0$, the corresponding domain is $-\pi / 2<\omega<\pi / 2$ and $0<\tau<\pi / 2-\omega$. This change of variables (48) is a conformal one.

In the new variables Eqs. $(46)$ and $(47)$ become ${ }^{* *}$

$$
\begin{aligned}
-\partial_{\tau}^{2} \phi+\partial_{\omega}^{2} \phi-\frac{\left(\partial_{\tau} \psi\right)^{2}-\left(\partial_{\omega} \psi\right)^{2}}{\phi^{3}}+\frac{\left(1-\phi^{2}\right) \phi}{\cos ^{2} \omega} & =0 \\
-\partial_{\tau} \frac{\partial_{\tau} \psi}{\phi^{2}}+\partial_{\omega} \frac{\partial_{\omega} \psi}{\phi^{2}}-\frac{2 \psi}{\cos ^{2} \omega} & =0 .
\end{aligned}
$$

Before solving these equations let us note that it is possible to predict the large- $t$ behavior of gauge field from the form of the conformal transformation (48). Indeed, the $t \rightarrow \infty$ limit corresponds to the line $\tau=\pi / 2-\omega$ on the $(\omega, \tau)$ plane. If one now takes the limit $|r-t| \rightarrow \infty$ (regardless of the limit for $|r-t| / t)$, the position on $(\omega, \tau)$ plane is either $\omega \rightarrow-\pi / 2, \tau \rightarrow 0$ or $\omega \rightarrow \pi / 2, \tau \rightarrow \pi$. This means that the entire line $\tau=\pi / 2-\omega$ corresponds to space-time points with finite differences between $r$ and $t$ and, therefore, if $\phi$ and $\psi$ are smooth functions of $\omega$ and $\tau$, then for asymptotic times the field is concentrated near the $r=t$ line. This corresponds to the fields expanding as a thin shell in space.

We must now supply Eqs. (49) with initial conditions, which are

$$
\begin{aligned}
\phi(\omega, \tau=0)^{2} & =1-\left(1-\kappa^{2}\right) \cos ^{2} \omega \\
\left.\partial_{\tau} \phi(\omega, \tau)\right|_{\tau=0} & =0 \\
\psi(\omega, \tau=0) & =0 \\
\left.\partial_{\tau} \psi(\omega, \tau)\right|_{\tau=0} & =\left.\frac{\rho}{1-\sin \omega} \partial_{t} \psi(\omega, \tau)\right|_{t=0} \\
& =\kappa\left(1-\kappa^{2}\right) \cos ^{2} \omega .
\end{aligned}
$$

\footnotetext{
** One can find a discussion of Eqs. (49) and some of its solutions in 21.
} 
One of the solutions of Eqs. (49), first found in 1977 by Lüscher [29] and Schechter [30], is

$$
\begin{aligned}
\phi(\omega, \tau)^{2} & =1-\left(1-q^{2}(\tau)\right) \cos ^{2} \omega \\
\psi(\omega, \tau) & =\frac{\dot{q}(\tau)}{2} \cos ^{2} \omega
\end{aligned}
$$

with a function $q(\tau)$ that satisfies

$$
\ddot{q}-2 q\left(1-q^{2}\right)=0 .
$$

This is the equation for a one-dimensional particle moving in double-well potential of the form $U(q)=(1-$ $\left.q^{2}\right)^{2} / 2$.

We now have to check that the Lüscher-Schechter solution satisfies the initial conditions, (50). This is indeed the case if one identifies $q(0)=\kappa$ and takes $\dot{q}(0)=0$. For the initial condition of this type (i.e. for energy $\varepsilon=\dot{q}^{2} / 2+U(q)<1 / 2$ ), the solution of Eq. (52) is

$$
q(\tau)=\tilde{q} \operatorname{dn}\left(\tilde{q}\left(\tau-\tau_{0}\right), k\right),
$$

where $d n$ is Jacobi's function and $\tilde{q}=\sqrt{2-\kappa^{2}}$ is the second stopping point for a particle in the potential $U(q)$. We have also defined

$$
k^{2}=2 \frac{1-\kappa^{2}}{2-\kappa^{2}} \quad \text { and } \quad \tau_{0} \tilde{q}=\frac{T}{2},
$$

where $T$, the period of oscillations in the potential $U(q)$, is $T=2 K(k)$, with $K(k)$ being the complete elliptic integral of the first kind. The idea is, of course, that "oscillations" in $\tau$ begin from the rest point, close to $\tau=0$.

Let us now look at several properties of the solution for large times. The solution (53) is apparently regular in the $(\omega, \tau)$ plane, and therefore for large times the field is concentrated near $r=t$. At asymptotic times the energy density, $e(r, t)$, is given by

$$
4 \pi e(r, t)=\frac{8 \pi}{g^{2} \rho^{2}}\left(1-\kappa^{2}\right)^{2}\left(\frac{\rho^{2}}{\rho^{2}+(r-t)^{2}}\right)^{3} .
$$

The change in topological charge is

$$
\begin{aligned}
\Delta Q & =\int_{0}^{\infty} d^{3} x d t \partial_{\mu} K^{\mu} \\
& =\frac{1}{2 \pi} \int d r d t\left[-\partial_{t}^{2} \psi+\partial_{r}^{2} \psi-\frac{2 \psi}{r^{2}}\right] \\
& =\frac{\pi}{2} \kappa\left(3-\kappa^{2}\right)-\operatorname{sign}(\kappa) \arccos \left(\frac{\operatorname{cn}(\tilde{q} \pi, k)}{\operatorname{dn}(\tilde{q} \pi, k)}\right) .
\end{aligned}
$$

The evolution of $\tilde{N}_{C S}$ begins from time $t=0$, where

$$
\tilde{N}_{C S}(0)=\frac{1}{4} \operatorname{sign}(\kappa)(1-|\kappa|)^{2}(2+|\kappa|),
$$

and as $t \rightarrow \infty$ its limit is $\tilde{N}_{C S}(\infty)=\tilde{N}_{C S}(0)+\Delta Q$.
We now estimate number of gluons produced by the described evolution. In $\phi, \psi$ language the chromoelectric and chromomagnetic fields are

$$
\begin{aligned}
E_{j}^{a}= & \frac{1}{r}\left(\partial_{t} \phi \sin \alpha-\frac{\partial_{r} \psi \cos \alpha}{\phi}\right) \Theta_{j}^{a} \\
& +\frac{1}{r}\left(\partial_{t} \phi \cos \alpha+\frac{\partial_{r} \psi \sin \alpha}{\phi}\right) \Pi_{j}^{a}+\frac{2 \psi}{r^{2}} \Sigma_{j}^{a},
\end{aligned}
$$

$$
\begin{aligned}
B_{j}^{a}= & -\frac{1}{r}\left(\partial_{r} \phi \cos \alpha+\frac{\partial_{t} \psi \sin \alpha}{\phi}\right) \Theta_{j}^{a} \\
& +\frac{1}{r}\left(\partial_{r} \phi \sin \alpha-\frac{\partial_{t} \psi \cos \alpha}{\phi}\right) \Pi_{j}^{a}+\frac{1-\phi^{2}}{r^{2}} \Sigma_{j}^{a} .
\end{aligned}
$$

Terms proportional to $\Sigma_{j}^{a}$ are longitudinal and die out as $t \rightarrow \infty$. The remainder is a purely transverse field. Now let us take into account that for $t \rightarrow \infty, \partial_{r} \phi \rightarrow \partial_{t} \phi$, and the same for $\psi$. Therefore

$$
\begin{aligned}
E_{j}^{a} \rightarrow & \frac{1}{r}\left(\partial_{r} \phi \sin \alpha-\frac{\partial_{r} \psi \cos \alpha}{\phi}\right) \Theta_{j}^{a} \\
& +\frac{1}{r}\left(\partial_{r} \phi \cos \alpha+\frac{\partial_{r} \psi \sin \alpha}{\phi}\right) \Pi_{j}^{a}, \\
B_{j}^{a} \rightarrow & -\frac{1}{r}\left(\partial_{r} \phi \cos \alpha+\frac{\partial_{r} \psi \sin \alpha}{\phi}\right) \Theta_{j}^{a} \\
& +\frac{1}{r}\left(\partial_{r} \phi \sin \alpha-\frac{\partial_{r} \psi \cos \alpha}{\phi}\right) \Pi_{j}^{a} .
\end{aligned}
$$

The main result becomes apparent when we choose a gauge where

$$
\phi \partial_{r} \phi \cos \alpha+\partial_{r} \psi \sin \alpha=0
$$

in which

$$
\begin{aligned}
E_{j}^{a} & \rightarrow \frac{1}{r} \sqrt{\frac{\left(\partial_{r} \psi\right)^{2}}{\phi^{2}}+\left(\partial_{r} \phi\right)^{2}} \Theta_{j}^{a} \\
& \rightarrow \frac{1-\kappa^{2}}{r \rho}\left(\frac{\rho^{2}}{\rho^{2}+(r-t)^{2}}\right)^{3 / 2} \Theta_{j}^{a}, \\
B_{j}^{a} & \rightarrow \frac{1-\kappa^{2}}{r \rho}\left(\frac{\rho^{2}}{\rho^{2}+(r-t)^{2}}\right)^{3 / 2} \Pi_{j}^{a} .
\end{aligned}
$$

We now perform a fourier transform, finding

$$
\begin{aligned}
& E_{j}^{a}(\vec{k})=4 \pi \rho\left(1-\kappa^{2}\right) K_{1}(\omega \rho) \Theta_{j}^{a} \\
& B_{j}^{a}(\vec{k})=4 \pi \rho\left(1-\kappa^{2}\right) K_{1}(\omega \rho) \Pi_{j}^{a},
\end{aligned}
$$

where $\Theta_{j}^{a}$ and $\Pi_{j}^{a}$ are the color/space projectors in momentum space analogous to those in coordinate space (2), the frequency $\omega=|\vec{k}|$, and $K_{1}$ is a Bessel function. One can easily verify that $B_{j}^{a}=\epsilon_{j l m} k_{l} E_{m}^{a} / k$, as is required for a radiation field. 


\section{EXPLOSIONS OF THE TURNING STATES: NUMERICAL STUDIES}

In the previous section we studied the asymptotic behavior of turning states constrained by sphaleron size and Chern-Simons number. In this section we consider step-by-step evolution of the turning states, a numerical analysis similar to sphaleron decay in electroweak theory [31,32]. The numerical approach naturally allows for mathematical flexibility, and it is used to consider the decay of static states which replace the unphysical power-law behavior of the fields at large distance with a phenomenologically more appropriate exponential tail. The classical field configurations are thus fixed in size indirectly by a mass parameter, a constraint which is subsequentially relaxed as the state quickly decays into free-streaming gluons.

As a result of scale invariance, the QCD instanton is of indeterminate size. While it is clear from phenomenology and lattice studies that the instanton vacuum favors a somewhat narrow size distribution centered at $\bar{\rho} \simeq 0.3$ $\mathrm{fm}$, the reason for this is yet unknown, although it is presumably due to interactions between instantons. It is thus natural that related classical objects, born in some way from the excitation of instantons, share a similar size. We arrange this by introducing a phenomenological gluon mass term in the initial configuration which is promptly relaxed as this unstable configuration begins to decay. We stress that the relaxation of this size constraint does not initiate the explosion; we will show that the turning state is an unstable configuration regardless of the mass term's presence.

The similarity between this procedure and electroweak sphaleron decay is clear, but not mathematically continuous. For the Higgs mechanism, the sphaleron size is constrained by the scalar vacuum condensate which introduces an effective mass for the gauge fields. This condensate vanishes at the sphaleron center, where the classical gauge field action is maximal. This feature persists in the limit of infinite gauge-Higgs coupling, when the Higgs field is fixed at its VEV constant for all other points in space. Inserting a mass term for the gauge fields by hand, as we do here for QCD, is thus very similar to this infinite coupling limit of electroweak dynamics, the only difference being that the mass is finite in all of space. This leads to a difference in field behavior at the origin.

We begin with the Yang-Mills action, with a phenomenological mass term added, and will look for static solutions with spherical symmetry in Minkowski space. The action is written:

$$
\begin{aligned}
S= & \frac{4 \pi}{g^{2}} \int d t d r\left\{\dot{\phi}_{1}^{2}+\dot{\phi}_{2}^{2}+\frac{1}{2} r^{2}{\dot{A_{1}}}^{2}-\left(\phi_{1}^{\prime}\right)^{2}-\left(\phi_{2}^{\prime}\right)^{2}\right. \\
& -\frac{\left(1-\phi_{1}^{2}-\phi_{2}^{2}\right)^{2}}{2 r^{2}}-2 A_{1}\left(\phi_{1} \phi_{2}^{\prime}-\phi_{1} \phi_{2}^{\prime}\right)
\end{aligned}
$$

$$
\left.-A_{1}^{2}\left(\phi_{1}^{2}+\phi_{2}^{2}\right)-m^{2}\left[\left(1+\phi_{1}\right)^{2}+\phi_{2}^{2}+\frac{1}{2} r^{2} A_{1}^{2}\right]\right\} .
$$

As before, we have taken a spherical ansatz similar to Witten's 19] for the gauge field. Following the notations of Eq. (11),

$$
A=\frac{1-\phi_{1}}{r}, \quad B=\frac{\phi_{2}}{r}, \quad C=A_{1}, \quad D=A_{0},
$$

where all scalar functions depend on both $r$ and $t$. We initially work in the temporal gauge, where $A_{0}(r, t)=0$.

The equations of motion are easily obtained, and we find

$$
\begin{aligned}
\ddot{\phi}_{1}- & \phi_{1}^{\prime \prime}+\frac{\phi_{1}}{r^{2}}\left(\phi_{1}^{2}+\phi_{2}^{2}-1\right)+\phi_{1} A_{1}^{2}-2 \phi_{2}^{\prime} A_{1} \\
& -\phi_{2} A_{1}^{\prime}+m^{2}\left(\phi_{1}+1\right)=0, \\
\ddot{\phi_{2}}- & \phi_{2}^{\prime \prime}+\frac{\phi_{2}}{r^{2}}\left(\phi_{1}^{2}+\phi_{2}^{2}-1\right)+\phi_{2} A_{1}^{2}+2 \phi_{1}^{\prime} A_{1} \\
& +\phi_{1} A_{1}^{\prime}+m^{2} \phi_{2}=0, \\
\ddot{A_{1}}+ & \frac{2}{r^{2}} A_{1}\left(\phi_{1}^{2}+\phi_{2}^{2}\right)+\frac{2}{r^{2}}\left(\phi_{1}^{\prime} \phi_{2}-\phi_{1} \phi_{2}^{\prime}\right) \\
& +m^{2} A_{1}=0 .
\end{aligned}
$$

In the static limit, we seek a purely magnetic turning state solution. One can be found for the $\phi_{1}$ field component from the equation

$$
\phi_{1}^{\prime \prime}-\frac{\phi_{1}}{x^{2}}\left(\phi_{1}^{2}-1\right)-\left(\phi_{1}+1\right)=0,
$$

which is simply Eqs. (66) with $\phi_{2}=A_{1}=0$ and written in terms of the dimensionless variable $x \equiv m r$. With the boundary conditions

$$
\begin{gathered}
\phi_{1}(x) \rightarrow 1 \text { as } x \rightarrow 0, \\
\phi_{1}(x) \rightarrow-1 \text { as } x \rightarrow \infty,
\end{gathered}
$$

A numerical solution is easily obtained and shown in Fig. 8. This is quite similar to the approximate electroweak solution found by Klinkhammer and Manton [18] in the limit of infinite Higgs self-coupling. The primary difference is the behavior near the origin, which in the QCD case involves a logarithm for $x \ll 1$ :

$$
\phi_{1}(x)=1+\frac{2}{3} x^{2} \ln x-\alpha x^{2}+\mathcal{O}\left(x^{4} \ln x\right),
$$

where $\alpha=1.98$ was determined numerically. Defining the turning state's size as the radius at which the profile is at half its maximum (i.e. where it crosses the origin), we find $m \rho \simeq 0.9$. We match this to the size of the average instanton and find

$$
m=0.9 \rho^{-1} \simeq 540 \mathrm{MeV} .
$$




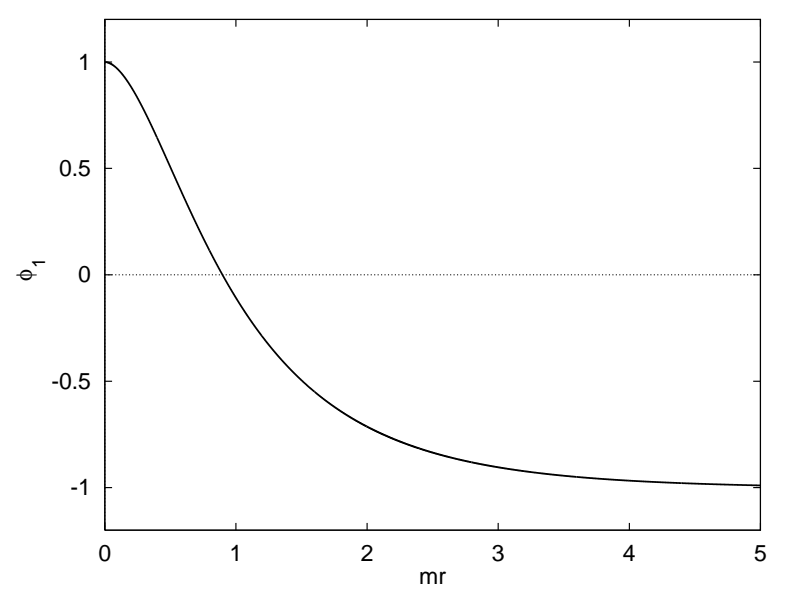

FIG. 8. The static turning state solution, $\phi_{1}(r, 0)$.

Before we discuss the decay of this configuration we must find the unstable modes orthogonal to it which determine the "downhill" directions in field space. This is done by solving eigenvalue equations for fluctuations in the fields $\phi_{2}$ and $A_{1}$ in the presence of the turning state configuration.

We take the terms linear in $\phi_{2}$ and $A_{1}$ from Eqs. (66) and require

$$
\ddot{\phi}_{2}(x, t)=-\omega^{2} \phi_{2}(x, t), \quad \ddot{A_{1}}(x, t)=-\omega^{2} A_{1}(x, t) .
$$

We then have the eigenvalue equations:

$$
\begin{aligned}
& \phi_{2}^{\prime \prime}+\left(\Omega^{2}-1+\frac{1}{x^{2}}\right) \phi_{2}+2 \phi_{1}^{\prime} A_{1}+\phi_{1} A_{1}^{\prime}=0, \\
& \left(\Omega^{2}-1-\frac{2}{x^{2}} \phi_{1}^{2}\right) A_{1}-\frac{2}{x^{2}}\left(\phi_{1}^{\prime} \phi_{2}-\phi_{1} \phi_{2}^{\prime}\right)=0,
\end{aligned}
$$

where $\phi_{1}$ is the classical solution in Fig. 8 and the dimensionless frequency is $\Omega=\omega / m$. The longitudinal field component may be eliminated with

$$
A_{1}=\frac{2\left(\phi_{1}^{\prime} \phi_{2}-\phi_{1} \phi_{2}^{\prime}\right)}{\left(\Omega^{2}-1\right) x^{2}-2 \phi_{1}^{2}}
$$

Substituting this into the first of Eqs. (71), we find the behavior near the origin:

$$
\phi_{2}(x)=c x^{\frac{1}{2}(1-\sqrt{1-8 y})},
$$

where

$$
y=\frac{1+\Omega^{2}}{1-\Omega^{2}}
$$

and $c$ is an arbitrary normalization. Both fields vanish as $x \rightarrow \infty$.

We have solved these equations numerically, finding the wave functions plotted in Fig. 9 with the frequency

$$
\omega^{2}=-3.4 m^{2} .
$$

The function $A_{1}$ is logarithmically divergent at the origin, reflecting the difference between this massive model and the electroweak case [33] in which the Higgs always vanishes at the origin ${ }^{\dagger \dagger}$. There is therefore no smooth continuation between this model and the electroweak in the limit of large coupling.

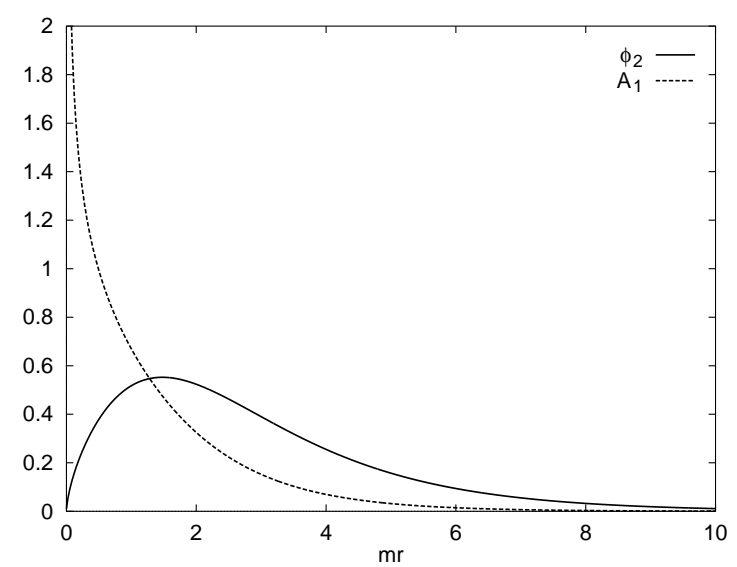

FIG. 9. The unstable eigenmodes, $\phi_{2}(r)$ and $A_{1}(r)$, arbitrarily normalized.

These solutions for the unstable modes, which along with the classical $\phi_{1}(r)$ complete our initial conditions, were put on a lattice with spacing $\Delta x=0.01$ and evolved at time steps of $\Delta \tau=5 \times 10^{-4}$, where $\tau \equiv m t$. The unstable modes, acting as a small push to properly initiate the decay, were normalized as

$$
\int d x\left(\phi_{2}(x)^{2}+2 x^{2} A_{1}(x)^{2}\right)=5 \times 10^{-3} .
$$

Coincident with this push we set $m=0$, in effect turning off the mass term, since here we are interested in the turning state decaying into the vacuum where no such term is motivated. Although this effectively removes the size constraint on $\phi_{1}(r, 0)$, the subsequent dynamical expansion is a result of the push in the unstable directions of this saddle-point solution rather than an inflation of the classical field configuration.

Once the real-time solutions to Eqs. (66) with $m=0$ have been found at a given time step, the total energy is readily computed as

$$
\begin{aligned}
E=\frac{4 \pi}{g^{2}} \int d r & {\left[\dot{\phi}_{1}^{2}+\dot{\phi}_{2}^{2}+\frac{1}{2} r^{2} \dot{A}_{1}^{2}+\left(\phi_{1}^{\prime}\right)^{2}+\left(\phi_{2}^{\prime}\right)^{2}\right.} \\
& +\frac{\left(1-\phi_{1}^{2}-\phi_{2}^{2}\right)^{2}}{2 r^{2}}-2 A_{1}\left(\phi_{1} \phi_{2}^{\prime}-\phi_{1} \phi_{2}^{\prime}\right)
\end{aligned}
$$

\footnotetext{
${ }^{\dagger}$ The dominant unstable mode in the electroweak as $\lambda \rightarrow \infty$ is the scalar field component orthogonal to the condensate [33. This mode is absent here.
} 


$$
\left.+A_{1}^{2}\left(\phi_{1}^{2}+\phi_{2}^{2}\right)\right]
$$

At every time step it was verified that the energy remains equal to that of the initial state. Taking the instanton vacuum value of

$$
\frac{8 \pi^{2}}{g^{2}}=12
$$

the total energy of the decaying object was calculated and found to be

$$
E=4.62 \rho^{-1} \simeq 2.8 \mathrm{GeV} .
$$

The Chern-Simons number was also computed at each time step. In our present gauge, this is written as

$$
N_{C S}=\frac{1}{2 \pi} \int d r\left[\left(1-\phi_{1}\right) \phi_{2}^{\prime}+\phi_{1}^{\prime} \phi_{2}-\left(1-\phi_{1}^{2}-\phi_{2}^{2}\right) A_{1}\right] .
$$

The gauge invariance of changes in this quantity were verified numerically.

The energy and Chern-Simons densities, defined as the integrands of Eqs. (76) and (79), are shown in Fig. 10. The shell-like expansion is illustrated in these plots, as well as the similarity of the energy profile at all times. Note that these plots are in a $1+1$ dimensional description, and the corresponding three-dimensional radial density differs by a factor of $1 / r^{2}$.

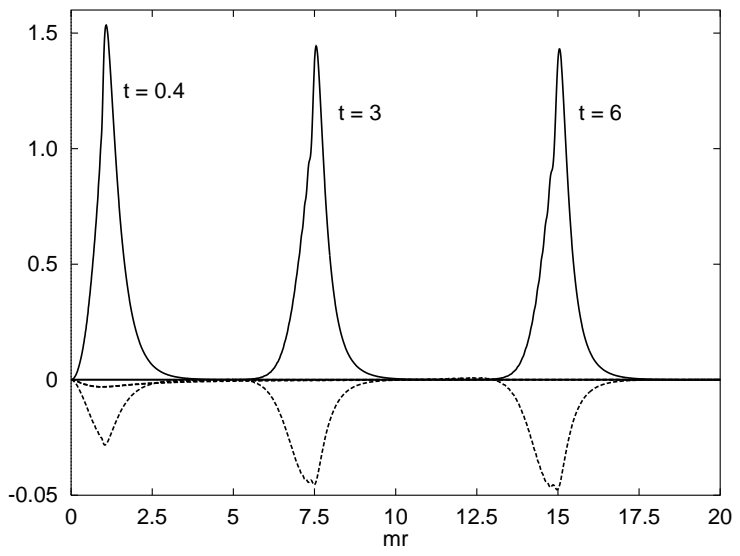

FIG. 10. Energy (solid lines) and Chern-Simons number densities (dashed lines) for three times during the explosion, $t=0.4,3$, and $6 \mathrm{fm}$. (Note the scale difference between the two quantities.)

It is clear from the curves in Fig. 10 that the ChernSimons number changes during the decay. As shown in Fig. 11, our fields stabilize at long times with $\Delta N_{C S} \simeq$ 0.12 . From this we see that the turning state does not complete an instanton transition, which would require a return to a state with integral Chern-Simons number. Due to this freezing in the topology, nontrivial fermionic solutions will accompany the resulting Yang-Mills fields. These will be discussed elsewhere.

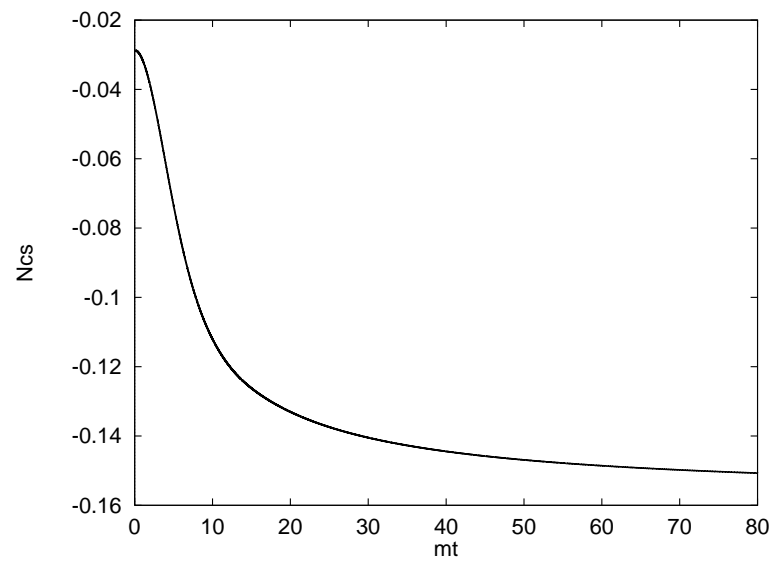

FIG. 11. Change in the Chern-Simons number.

The transition from a purely magnetic configuration to one with equal electric and magnetic components is shown in Fig. 12, for an early and late time in the evolution. The decay progresses rather rapidly; at $t=1.4 \mathrm{fm}$, the ratio $E(r)^{2} / B(r)^{2} \simeq 0.95$ for all $r$. Thereafter the ratio continues to quickly approach unity.

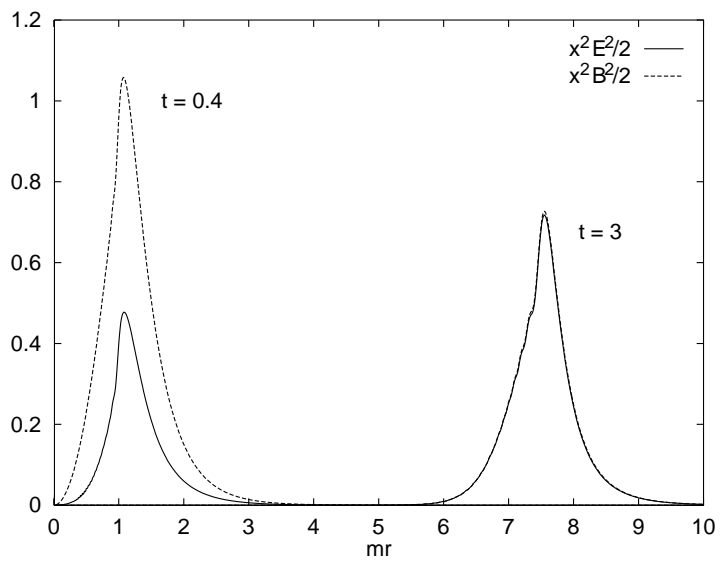

FIG. 12. The electric (solid lines) and magnetic (dashed lines) fields at times $t=0.4 \mathrm{fm}$ and $t=3 \mathrm{fm}$. Plotted are $\frac{1}{2} x^{2} E(x)^{2}$ and $\frac{1}{2} x^{2} B(x)^{2}$; their sum is the energy density.

In order to analyze the final state at late times, we we work in a gauge in which $B(r, t)=0$. This requires the transformation to a new set of fields:

$$
\begin{aligned}
& \tilde{\phi}_{1}=\phi_{1} \cos \theta+\phi_{2} \sin \theta \\
& \tilde{\phi}_{2}=-\phi_{1} \sin \theta+\phi_{2} \cos \theta \\
& \tilde{A}_{1}=A_{1}-\theta^{\prime} \\
& \tilde{A}_{0}=-\dot{\theta},
\end{aligned}
$$

where

$$
\theta=\arctan \left(\frac{\phi_{2}}{\phi_{1}}\right)
$$

Promptly dropping the tildes, we write the total energy in terms of the new fields, 


$$
\begin{aligned}
E= & \frac{4 \pi}{g^{2}} \int d r\left[\dot{\phi}_{1}^{2}+\left(\phi_{1}^{\prime}\right)^{2}+\frac{\left(1-\phi_{1}^{2}\right)^{2}}{2 r^{2}}\right. \\
& \left.+\frac{r^{2}}{2}\left(\dot{A_{1}}-A_{0}^{\prime}\right)^{2}+\phi_{1}^{2}\left(A_{1}^{2}+A_{0}^{2}\right)\right] .
\end{aligned}
$$

At late times, the field strength is confined to a thin shell at radius $r=t$. Free, expanding field behavior is also observed numerically, such that

$$
\dot{\Phi}(r, t)=-\Phi^{\prime}(r, t)
$$

for each field $\phi_{1}, A_{1}$, and $A_{0}$. This simplifies the equations of motion for the latter two,

$$
\begin{aligned}
& \ddot{A_{1}}-\dot{A_{0}}{ }^{\prime}=-\frac{2}{r^{2}} \phi_{1}^{2} A_{1} \\
& A_{0}^{\prime \prime}-\dot{A}_{1}{ }^{\prime}=-\frac{2}{r}\left(A_{0}^{\prime}-\dot{A_{1}}\right)+\frac{2}{r^{2}} \phi_{1}^{2} A_{1},
\end{aligned}
$$

in that the right hand sides of both vanish as the shell expands at large times. We can thus conclude that

$$
\dot{A_{1}}-A_{0}^{\prime}=0
$$

for $m t \gg 1$.

The condition for this gauge is

$$
\dot{A_{0}} \phi_{1}+2 A_{0} \dot{\phi}_{1}-A_{1}^{\prime} \phi_{1}-2 A_{1} \phi_{1}^{\prime}=0 .
$$

Combining this with Eq. (85), we have

$$
\phi_{1}\left(\dot{A_{0}}-A_{1}^{\prime}\right)-2\left(\phi_{1}^{\prime} A_{1}-\dot{\phi_{1}} A_{0}\right)=0,
$$

and can deduce that

$$
A_{0}+A_{1}=0
$$

at late times.

The contribution from $\phi_{1}$ will be of the form

$$
\phi_{1}(r, t)=1+\varphi(r, t),
$$

where $\varphi$ is an excitation above the vacuum. Using the result just obtained (88), its linearized equation of motion simplifies to

$$
\ddot{\varphi}-\varphi^{\prime \prime}+\frac{2}{r^{2}} \varphi=0 .
$$

The solution is of the form

$$
\varphi(r, t)=\int d k \varphi(k) r\left[j_{1}(k r)+i y_{1}(k r)\right] \cos (k t),
$$

with fourier amplitudes $\varphi(k)$ and the spherical Bessel functions $j_{1}(z)$ and $y_{1}(z)$.

Although the majority of gluon radiation is carried in the $\phi_{1}$ field, physical quanta also lie in small excitations of the field

$$
\psi(x, \tau)=\phi_{1}(x, \tau) A_{0}(x, \tau),
$$

which encodes oscillations between the transverse degrees of freedom. From Eqs. (86) and (88), we have a wave equation,

$$
\ddot{\psi}-\psi^{\prime \prime}=0 .
$$

These harmonics contribute to the energy via the final term in Eq. (82).

The total energy can then be written in momentum space as

$$
E=\frac{16 m}{g^{2}} \int d p\left[p^{2} \varphi(p)^{2}+\psi(p)^{2}\right],
$$

in terms of the dimensionless momentum $p=k / m$. The fourier amplitudes are computed from the solutions of the spatial fields:

$$
\begin{aligned}
\varphi(p)^{2} & =\left|\int d x p x\left[j_{1}(p x)+i y_{1}(p x)\right] \varphi(x)\right|^{2} \\
\psi(p)^{2} & =\left|\int d x e^{i p x} \psi(x)\right|^{2} .
\end{aligned}
$$

Numerically, this expression for the energy is within $1 \%$ of that of the initial configuration for all times $t \geq 3 \mathrm{fm}$, further demonstrating the rapid onset of free-field behavior.

\section{PRODUCTION OF TURNING STATES IN HEAVY ION COLLISIONS}

\section{A. The pQCD Cutoff in Vacuum Versus Excited Matter}

Although this paper does not generally deal with phenomenological applications, we will eventually come to an estimate of the number of gluons produced in the explosion of a turning state. The resulting divergence in this number cannot be resolved without some explanation of the limited applicability of the classical Yang-Mills description. This leads to the issue of the pQCD cutoff.

It is well known that in the $\mathrm{QCD}$ vacuum the "semihard" or "substructure scale" $Q^{2} \sim 1-2 \mathrm{GeV}^{2}$ is simultaneously the lower boundary of pQCD as well as the upper boundary of low energy effective approaches such as chiral Lagrangians. Furthermore, in any discussion of instanton-induced reactions it is implicitly assumed that instantons were the primary source of that scale, and since they are included explicitly no other nonperturbative cutoffs are needed. This, of course, is not strictly true as confining forces require the final state be comprised of hadrons, but we make the usual separation of scales and assume that final state interactions merely redistribute the wave functions without changing the total probabilities. 
We assert, however, that the pQCD cutoff is quite different in heavy ion collisions. It has been argued over the years that excited matter might be in a QuarkGluon Plasma (QGP) phase of QCD (see e.g. Ref. [34). Whether equilibrated or not, it is nevertheless qualitatively very different from the $\mathrm{QCD}$ vacuum: instantons are suppressed, and there is neither confinement nor chiral symmetry breaking to set a nonperturbative cutoff.

Therefore the limits on Yang-Mills field description are entirely different, and actually determined by much simpler phenomena. The QGP, a plasma-like phase, screens itself perturbatively [34]. A quasi-particle description becomes appropriate, in which the quarks and gluons have finite effective masses. In equilibrium and at high temperature these are "thermal masses"; the gluon has the well-known effective mass 34

$$
M_{g}^{2}=\frac{g^{2} T^{2}}{2}\left(\frac{N_{c}}{3}+\frac{N_{f}}{6}\right)
$$

where $N_{c}$ and $N_{f}$ are the number of colors and flavors, respectively. Although this mass grows with temperature at high $T$, just above $T_{c}$ it is actually smaller than the pQCD cutoff in vacuum. Such non-monotonic behavior is confirmed by lattice thermodynamics data, which can be well fitted with quasiparticle masses.

Moreover, in the "RHIC window", $T_{c}<T<3 T_{c}$, one finds the approximately constant gluon and quark effective masses [35]

$$
M_{g} \approx .4 \mathrm{GeV}, \quad M_{q} \approx .3 \mathrm{GeV},
$$

the first of which provides the cutoff of our classical treatment; at this scale the classical Yang-Mills action for gluons is to be modified by inclusion of an appropriate effective Lagrangian describing such screening effects, such as those suggested by Taylor and Wong [36].

\section{B. Multiplicity and Spectra of Prompt Gluons}

With solutions for the fields at all times, in both the analytic and numerical treatments of the previous two sections, we have analyzed the final states to determine the particle number. While we find a similar number of produced particles from both approaches, about four gluons produced per turning state, the energy distribution of this prompt glue is very sensitive to the classical configuration used to initiate the explosion. While the field solutions found using constrained quantization (Section III) and an effective mass (Section V) are qualitatively similar, both leading to an expanding shell of radiation, we find a substantial difference in final state momentum distributions. This can be traced to the details of the two solutions as $r \rightarrow \infty$, where we contrast a power-law behavior in Eq. (36) with an exponential fall-off in the solution of Fig. 8, as $\phi_{1}(r, 0) \sim \exp (-m r)-1$. As mentioned above, we consider the second case to be of greater physical relevance, as the gluonic field is not massless phenomenologically (and even vacuum instantons ought to have exponential tails). We now consider both results.

To find the number of gluons in each mode one compares the field strength in the momentum representation to those which have energy $\omega$. In the evolution described analytically in Section IV, the occupation number is

$$
\nu(\vec{k})=\frac{64 \pi^{2}}{g^{2}}\left(1-\kappa^{2}\right)^{2} \frac{\rho^{2}}{\omega} K_{1}(\omega \rho)
$$

and the gluon energy distribution function is

$$
n(\omega)=\frac{32}{g^{2}}\left(1-\kappa^{2}\right)^{2} \omega \rho^{2} K_{1}^{2}(\omega \rho)
$$

The corresponding energy spectrum $E(\omega)=\omega n(\omega)$ is shown in Fig. 13.

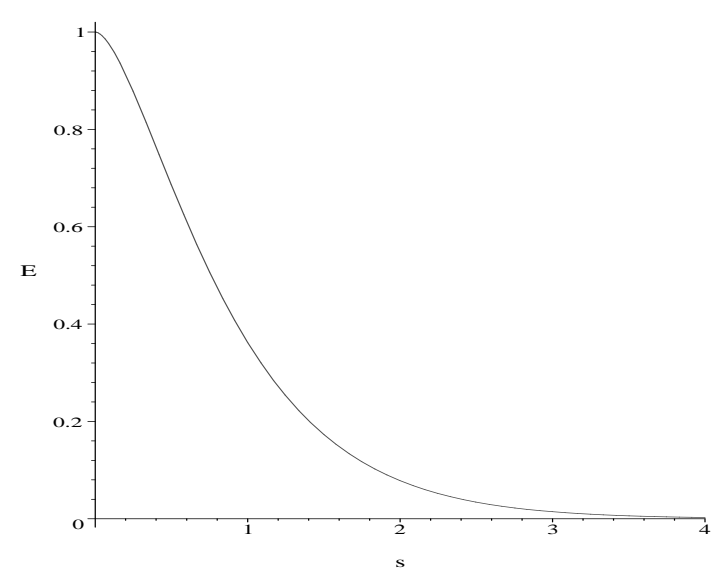

FIG. 13. The energy spectrum $E(\omega)$ obtained from the analytic solution, in units of $\frac{16}{g^{2}}\left(1-\kappa^{2}\right)^{2}$, versus $s=\omega \rho$.

Because the fourier transforms of the fields are finite, the occupation number (97) behaves as $1 / \omega$ for small $\omega$. The number of particles is thus logarithmically divergent and should be cutoff at some low scale where the pQCD description of gluons is no longer valid, leading to $N_{g} \sim \log \left(\frac{1}{M_{g} \rho}\right)$. As explained in the previous Subsection, in heavy ion collisions this cutoff, identified with the gluon effective mass of Eq. (96), is still relatively small as compared to the typical momenta of gluons produced.

The prompt particle energy distribution was also obtained in the numerical treatment of Section V, defined as the integrand of the expression in Eq. (93), is shown in Fig. 14 at a very late time in the evolution $(\tau=50$ or $t \simeq 20 \mathrm{fm}$ ). Like the previous distribution, it is finite at the origin, but in contrast it peaks at nonzero momentum. For illustrative purposes it is compared with a thermal distribution of bosons at a temperature $T=285$ $\mathrm{MeV}$. We note that in an equilibrated environment, the effective mass and screening effects will only modify this profile below $k=0.4 \mathrm{GeV}$, where a relatively small part of the spectrum resides. 
The produced gluons are free streaming, with no mechanism for equilibration, and yet our distribution is very similar to the thermal one for momenta below about 1.5 $\mathrm{GeV}$. Such a nearly thermal distribution has also been obtained from similar calculations using an entirely different classical field approach in Ref. [37]. One can speculate that such an ostensible equilibration may contribute to the the success of hydrodynamics in calculating particle spectra and elliptic flow at RHIC [38]. Our finding of four physical gluons from every decayed turning state is also in line with RHIC entropy production, assuming that the density of these classical objects corresponds to that of instantons in the vacuum. The total energy of the turning state, found above to be $2.8 \mathrm{GeV}$, is carried by gluons with a distribution peaked around $800 \mathrm{MeV}$. This average can be viewed as an upper bound, since in a more complete treatment a portion of this energy will be used for the production of light quark pairs. This next step will be addressed in a later publication.

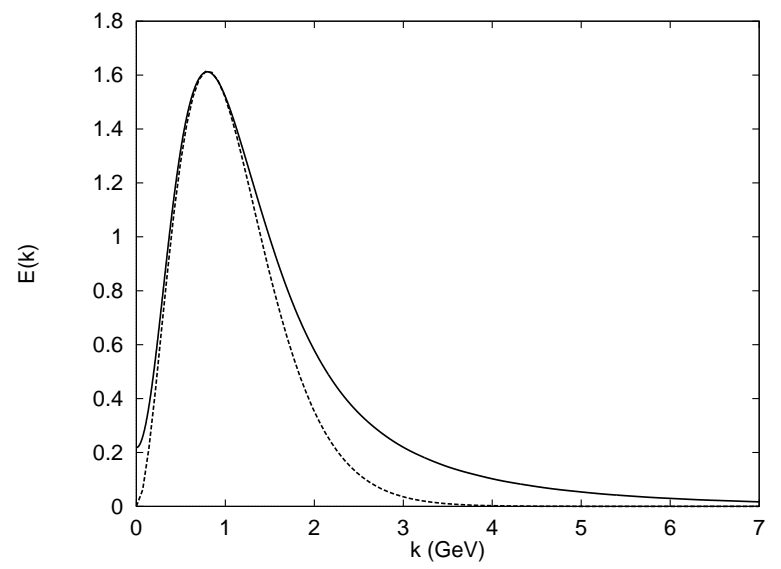

FIG. 14. Energy spectrum of prompt gluons (solid line), obtained from the numerical solution, and a thermal distribution with $T=285 \mathrm{MeV}$ (dashed line).

\section{CONCLUSIONS}

In this work we have studied forced tunneling in pure SU(2) Yang-Mills theory. This process, generated through the excitation of instantons in the QCD vacuum, leads to unstable classical turning states which explosively decay into gluonic radiation. These states and their decays are similar to the physics of sphalerons in the electroweak theory.

If this semi-classical treatment of pure Yang-Mills theory is indeed relevant to the physics of QCD, the turning states should play a prominent role in the production of glue in high-energy hadronic collisions. In the case of $N N$ collisions the produced gluons will propagate into the QCD vacuum, to be quickly recombined into secondary hadrons. For heavy ion collisions, however, the large quantity of prompt glue produced from the many turning states would be released into a highly excited, perhaps deconfined medium. Observable consequences in both situations were discussed recently in Ref. [39], and the results of this work support many of the estimates therein. Finally, we have obtained energy spectra for the prompt gluons that serve as the initial state in the dynamics of a heavy ion collision and found that, unlike the overall explosive dynamics, it is sensitive to the details of the initial turning state profile. We plan to further investigate phenomenological implications of the turning states in later works, with the role of fermion production a top priority.

Acknowledgements: We thank I. Zahed and R. Venugopalan for useful discussions. This work is partially supported by the US-DOE grants Nos. DE-FG0288ER40388 and DE-FG03-97ER41014.

[1] A. A. Belavin, A. M. Polyakov, A. S. Shvarts, and Y. S. Tyupkin, Phys. Lett. B 59, 85 (1975).

[2] G. 't Hooft, Phys. Rev. D 14, 3432 (1976) [Erratum-ibid. D 18, 2199 (1976)].

[3] E. V. Shuryak, Nucl. Phys. B 203, 93 (1982); D. Diakonov and V. Y. Petrov, Nucl. Phys. B 272, 457 (1986).

[4] T. Schafer and E. V. Shuryak, Rev. Mod. Phys. 70, 323 (1998).

[5] V. A. Kuzmin, V. A. Rubakov, and M. E. Shaposhnikov, Phys. Lett. B 155, 36 (1985); P. Arnold and L. D. McLerran, Phys. Rev. D 36, 581 (1987).

[6] A. Ringwald, Nucl. Phys. B 330, 1 (1990); O. Espinosa, Nucl. Phys. B 343, 310 (1990); V. I. Zakharov, Nucl. Phys. B 353, 683 (1991); M. Maggiore and M. A. Shifman, Phys. Rev. D 46, 3550 (1992); D. Diakonov and V. Petrov, Phys. Rev. D 50, 266 (1994).

[7] V. V. Khoze and A. Ringwald, Phys. Lett. B 259, 106 (1991);

[8] S. Moch, A. Ringwald, and F. Schrempp, Nucl. Phys. B 507, 134 (1997).

[9] A. Ringwald and F. Schrempp, Phys. Lett. B 503, 331 (2001); F. Schrempp, arXiv:hep-ph/0109032.

[10] D. E. Kharzeev, Y. V. Kovchegov, and E. Levin, Nucl. Phys. A 690, 621 (2001).

[11] M. A. Nowak, E. V. Shuryak, and I. Zahed, Phys. Rev. D 64, 034008 (2001).

[12] G. W. Carter, D. M. Ostrovsky, and E. V. Shuryak, Phys. Rev. D 65, 074034 (2002).

[13] E. A. Kuraev, L. N. Lipatov, and V. S. Fadin, Sov. Phys. JETP 45 (1977) 199 [Zh. Eksp. Teor. Fiz. 72 (1977) 377]; I. I. Balitsky and L. N. Lipatov, Sov. J. Nucl. Phys. 28, 822 (1978) [Yad. Fiz. 28, 1597 (1978)]; L. N. Lipatov, Sov. Phys. JETP 63, 904 (1986) [Zh. Eksp. Teor. Fiz. 90, 1536 (1986)].

[14] L. D. McLerran and R. Venugopalan, Phys. Rev. D 49, 2233 (1994); L. D. McLerran and R. Venugopalan, Phys. Rev. D 49, 3352 (1994); L. D. McLerran and R. Venugopalan, Phys. Rev. D 50, 2225 (1994); A. Krasnitz and 
R. Venugopalan, Phys. Rev. Lett. 84, 4309 (2000).

[15] D. Kharzeev and E. Levin, Nucl. Phys. B 578, 351 (2000).

[16] E. V. Shuryak, Phys. Lett. B 486, 378 (2000).

[17] N. S. Manton, Phys. Rev. D 28, 2019 (1983);

[18] F. R. Klinkhamer and N. S. Manton, Phys. Rev. D 30, 2212 (1984).

[19] E. Witten, Phys. Rev. Lett. 38, 121 (1977).

[20] T. Akiba, H. Kikuchi, and T. Yanagida, Phys. Rev. D 38, 1937 (1988).

[21] E. Farhi, V. V. Khoze, and R. J. Singleton, Phys. Rev. D 47, 5551 (1993).

[22] C. G. Callan, R. F. Dashen, and D. J. Gross, Phys. Rev. D 17, 2717 (1978).

[23] A. V. Yung, Nucl. Phys. B 297, 47 (1988).

[24] T. Schafer and E. V. Shuryak, Phys. Rev. D 53, 6522 (1996)

[25] E. V. Shuryak, Nucl. Phys. B 302, 574 (1988).

[26] E. V. Shuryak, Nucl. Phys. B 302, 621 (1988).

[27] J. J. Verbaarschot, Nucl. Phys. B 362, 33 (1991) [Erratum-ibid. B 386, 236 (1991)].

[28] A. V. Smilga, Nucl. Phys. B 459, 263 (1996).

[29] M. Lüscher, Phys. Lett. B 70, 321 (1977).

[30] B. M. Schechter, Phys. Rev. D 16, 3015 (1977).

[31] M. Hellmund and J. Kripfganz, Nucl. Phys. B 373, 749 (1992).

[32] J. Zadrozny, Phys. Lett. B 284, 88 (1992).

[33] T. Akiba, H. Kikuchi, and T. Yanagida, Phys. Rev. D 40, 588 (1989).

[34] E. V. Shuryak, Phys. Rept. 61, 71 (1980).

[35] P. Levai and U. W. Heinz, Phys. Rev. C 57, 1879 (1998).

[36] J. C. Taylor and S. M. Wong, Nucl. Phys. B 346, 115 (1990).

[37] A. Krasnitz and R. Venugopalan, Phys. Rev. Lett. 86, 1717 (2001).

[38] D. Teaney, J. Lauret, and E. V. Shuryak, arXiv:nuclth/0110037.

[39] E. V. Shuryak, Phys. Lett. B 515, 359 (2001). 